\title{
三组分反应高效合成螺[环戊烷-1,3'-吲哚啉]衍生物
}

\author{
马蔚青韩莹* 孙晶颜朝国* \\ (扬州大学化学化工学院 江苏扬州 225002)
}

\begin{abstract}
摘要 烷基异氰(酸酯、丁炔二酸二酯(丙炔酸酯)和 3-苯甲酰亚甲基-2-吲哚酮在甲苯中回流反应，高产率地生成螺[环戊 烷-1,3'-吲哚啉]衍生物. 然而, 含有游离氨基的 3-苯甲酰亚甲基-2-吲哚酮参加反应时, 未取代的氨基可与另一分子丁炔 二酸二酯及烷基异氧酸酯继续反应形成含有氨基取代的氮杂-1,3-二烯支链的螺[环戊烷-1,3'-吲哚啉]衍生物. 另一方面, 3-芳亚甲基吲哚-2-酮参与三组分反应时, 仅有游离氨基参与反应, 生成 2-(2-氧吲梠-1-基)-3-[(烷基亚氨基)亚甲基]丁酸 酯. 发现两种游离氨基参与多组分反应时, 分别形成了含有 $\mathrm{C} 2$-取代的 1-氮杂-1,3-丁二烯 $(\mathrm{C}=\mathrm{C}-\mathrm{C}=\mathrm{NR})$ 和 $\mathrm{C} 4$-取代的 1-氮杂-1,2-丁二烯 $(\mathrm{C}-\mathrm{C}=\mathrm{C}=\mathrm{NR})$ 结构单元的吲哚酮衍生物.
\end{abstract}

关键词 异氰酸酯; 丁炔二酸二酯; 3-苯甲酰亚甲基吲哚酮; 螺[环戊烷-1,3'-吲哚啉]; 多组分反应

\section{Three-Component Reaction for Efficient Synthesis of Functionalized Spiro[cyclopentane-1,3'-indolines]}

\author{
Ma, Weiqing Han, Ying* Sun, Jin Yan, Chaoguo* \\ (College of Chemistry \& Chemical Engineering, Yangzhou University, Yangzhou, Jiangsu 225002)
}

\begin{abstract}
The three-component reaction of alkylisocyanide, dialkyl acetylenedicarboxylate (alkyl propiolate) and 3-phenacylideneoxindole in refluxing toluene afforded the functionalized spiro[cyclopentane-1,3'-indolines] in satisfactory yields. However, the similar reaction of 3-phenacylideneoxindoles with unsubstituted NH group resulted in spiro[cyclopentane-1,3'-indolines] with additional aza-buta-1,3-diene scaffold. On the other hand, the three-component reactions with 3-arylideneoxindolin2-ones only gave 2-(2-oxoindolin-1-yl)-3-((alkylimino)methylene)succinates. It was also interesting to find that the reaction of free amino group in two kinds of functionalized 3-methyleneoxindoles with alkylisocyanide and dialkyl acetylenedicarboxylate selectively gave $\mathrm{C}$-substituted 1-aza-buta-1,3-diene $(\mathrm{C}=\mathrm{C}-\mathrm{C}=\mathrm{NR})$ or $\mathrm{C}$-substituted 1-aza-buta-1,2-diene $(\mathrm{C}-\mathrm{C}=\mathrm{C}=$ NR) scaffold in the molecules.

Keywords isocyanide; dialkyl acetylenedicarboxylate; 3-phenacylideneoxindole; spiro[cyclopentane-1,3'-indoline]; multicomponent reaction
\end{abstract}

\section{Introduction}

The spirooxindole is one of the most privileged heterocyclic rings, which not only exists in several naturally occurring substances, but also is featured in many medicinally relevant compounds with wide applications as antimicrobial and antitumor agents and inhibitors of the human neurokinin receptors. ${ }^{[1]}$ Additionally, the construction of diverse spirooxindoles is often accompanied with interesting regio-, diastereo- and enantio-selectivity. ${ }^{[2]}$

These properties have prompted many efforts toward the multilateral investigations of various spirooxindoline de- rivatives. Consequently, the considerable works have been paid to develop efficient synthetic methodologies for the diversely structural spirooxindoles. ${ }^{[3]}$

Isocyanide is a functional group with outstanding reactivity, including the ability to react with both electrophiles and nucleophiles simultaneously. ${ }^{[4]}$ As a matter of fact, the unique features of alkylisocyanide make them versatile synthons for the synthesis of the divergent types of heterocycles. ${ }^{[5]}$ Furthermore, their compatibility with domino and cascade reactions has paved the way for developing novel atom and bond economic synthetic strategies. ${ }^{[6]}$ Isocyanide-based multicomponent reactions are powerful

\footnotetext{
* Corresponding authors. E-mail: cgyan@yzu.edu.cn; hanying@yzu.edu.cn Received March 21, 2021; revised April 14, 2021; published online May 13, 2021.

Project supported by the National Natural Science Foundation of China (No. 21572196) and the Priority Academic Program Development of Jiangsu Higher Education Institutions.
}

国家自然科学基金(No. 21572196)和江苏高校优势学科建设工程资助项目. 
tools for the synthesis of a wide variety of organic compounds. ${ }^{[7]}$ Passerine reaction, Ugi reaction and Van Leusen reaction are the most famous synthetic reactions by using isocyanide as key substrate. ${ }^{[8]}$ In this respect, the addition of isocyanide to electron-deficient alkyne resulted in active Huisgen' 1,4-dipole, which can be trapped by various electrophiles and nucleophiles to give versatile acyclic and heterocyclic compounds. ${ }^{[9]}$ Therefore, potential applications of the multicomponent reaction containing alkylisocyanide and electron-deficient alkynes have attracted significant attention in organic synthesis. ${ }^{[10]}$ In recent years, many domino or multicomponent reactions based on the nucleophilic addition of alkylsocyanide and electron-deficient alkynes have been successfully developed. ${ }^{[11-13]}$ In continue our aim to develop more efficient protocols for diverse spirooxindoles, ${ }^{[14-15]}$ herein we wish to report the simple and efficient synthesis of spiro[cyclopentane-1,3'-indoline] derivatives by three-component reaction of alkylisocyanides, electron-deficient alkynes and 3-methyleneoxindoles.

\section{Results and discussion}

Initially, the reaction conditions were briefly examined by using cyclohexylisocyanide, dimethyl acetylenedicarboxylate (DMAD) and 3-phenacylideneoxindole as standard reaction (Table 1). The three-component reaction in methylene dichloride, acetonitrile and toluene gave the spiro compound $\mathbf{1 b}$ in $34 \%, 36 \%$ and $42 \%$ yields, respectively (Entries 1 3). However, no spiro compound was formed when the reaction was carried out in tetrahydrofuran, methanol and ethanol (Entries $4 \sim 6$ ). The reaction in refluxing tetrahydrofuran (THF) still did not produce the spiro compound (Entry 7). When the reaction was carried out in acetonitrile and toluene at the elevated temperature, the yields of spiro compound $\mathbf{1 b}$ increased to $45 \%$ and $70 \%$ (Entries 8,9). The reaction in refluxing toluene for $0.5 \mathrm{~h}$ gave the product $\mathbf{1 b}$ in $86 \%$ yield (Entry 10 ). When the reaction was carried in refluxing toluene for $2 \mathrm{~h}$, the yield of $\mathbf{1 b}$ remained as $83 \%$ (Entry 11). Thus, the best reaction conditions were carrying the reaction in refluxing toluene for $0.5 \mathrm{~h}$.

With the optimized reaction conditions in hand, the scope of the substrates was developed. The results are summarized in Table 2. Both cyclohexylisocyanide and $t$-butylisocyanide were successfully used in the reaction. Various 3-phenacylideneoxindoles reacted smoothly to give the spiro[cyclopentane-1,3'-indolines] $\mathbf{1 a} \sim \mathbf{1 i}$ in satisfactory yields. The substituents showed marginal effect on the yields. On the other hand, the reaction with ethyl or 2-(2-oxoindolin-3-ylidene)acetates proceeded smoothly to give the expected spiro compounds $\mathbf{1} \mathbf{j} \sim \mathbf{1} \mathbf{m}$ in good yields. These results showed that this three-component reaction has a widely variety of substrates. The substituents on the alkylisocyanides and 3-methyleneoxindoles showed marginal effect on the yields. The chemical structures of the spiro compounds $\mathbf{1} \mathbf{a} \sim \mathbf{1} \mathbf{m}$ were fully characterized by
Table 1 Optimizing reaction conditions ${ }^{a}$<smiles>COC(=O)C#C[C+]1CCCCC1C#N</smiles>

\begin{tabular}{ccccc}
\hline Entry & Solvent & Temp./ & Time/h & Yield $^{b} \%$ \\
\hline 1 & $\mathrm{CH}_{2} \mathrm{Cl}_{2}$ & r.t. & 12 & 34 \\
2 & $\mathrm{MeCN}$ & r.t. & 12 & 36 \\
3 & $\mathrm{PhMe}$ & r.t. & 12 & 42 \\
4 & $\mathrm{THF}$ & r.t. & 12 & - \\
5 & $\mathrm{EtOH}$ & r.t. & 12 & - \\
6 & $\mathrm{MeOH}$ & r.t. & 12 & - \\
7 & $\mathrm{THF}$ & 60 & 12 & - \\
8 & $\mathrm{MeCN}$ & 60 & 6 & 45 \\
9 & $\mathrm{PhMe}$ & 60 & 2 & 70 \\
$\mathbf{1 0}$ & $\mathbf{P h M e}$ & $\mathbf{1 1 0}$ & $\mathbf{0 . 5}$ & $\mathbf{8 6}$ \\
11 & $\mathrm{PhMe}$ & 110 & 2 & 83 \\
\hline
\end{tabular}

$a$ Reaction conditions: alkylisocyanide $(0.45 \mathrm{~mol})$, DMAD $(0.45 \mathrm{mmol})$, 3-methyleneoxindole $(0.3 \mathrm{mmol})$, solvent $(10.0 \mathrm{~mL}) .{ }^{b}$ Isolated yields

spectroscopy and were confirmed by the determination of the single crystal structure of the compounds $\mathbf{1 b}$ (CCDC 2059918) and 1f (CCDC 2059919) (Figure 1).

Table 2 Synthesis of spiro[cyclopentane-1,3'-indolines] 1a $\sim$ $1 \mathbf{m}^{a}$

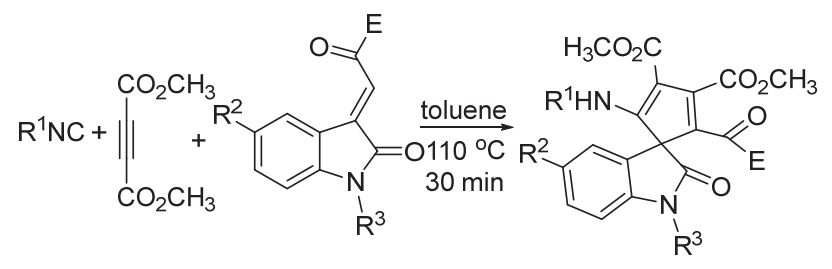

\begin{tabular}{clclllc}
\hline Compd. & \multicolumn{1}{c}{$\mathrm{R}^{1}$} & $\mathrm{R}^{2}$ & $\mathrm{R}^{3}$ & \multicolumn{1}{c}{$\mathrm{E}$} & $\mathrm{Yield}^{b} / \%$ \\
\hline $\mathbf{1 a}$ & Cyclohexyl & $\mathrm{H}$ & $\mathrm{Bn}$ & $p-\mathrm{CH}_{3} \mathrm{C}_{6} \mathrm{H}_{5}$ & 82 \\
$\mathbf{1 b}$ & Cyclohexyl & $\mathrm{Cl}$ & $\mathrm{Bn}$ & $p-\mathrm{CH}_{3} \mathrm{OC}_{6} \mathrm{H}_{5}$ & 86 \\
$\mathbf{1 c}$ & Cyclohexyl & $\mathrm{Cl}$ & $\mathrm{Bn}$ & $p-\mathrm{CH}_{3} \mathrm{C}_{6} \mathrm{H}_{5}$ & 82 \\
$\mathbf{1 d}$ & Cyclohexyl & $\mathrm{F}$ & $\mathrm{Bn}$ & $p-\mathrm{CH}_{3} \mathrm{OC}_{6} \mathrm{H}_{5}$ & 78 \\
$\mathbf{1 e}$ & Cyclohexyl & $\mathrm{F}$ & $\mathrm{Bn}$ & $p-\mathrm{CH}_{3} \mathrm{C}_{6} \mathrm{H}_{5}$ & 75 \\
$\mathbf{1 f}$ & $\mathrm{Cyclohexyl}$ & $\mathrm{F}$ & $n-\mathrm{Bu}$ & $\mathrm{C}_{6} \mathrm{H}_{5}$ & 62 \\
$\mathbf{1 g}$ & $t$ - $\mathrm{Bu}$ & $\mathrm{Cl}$ & $\mathrm{Bn}$ & $p-\mathrm{CH}_{3} \mathrm{OC}_{6} \mathrm{H}_{5}$ & 85 \\
$\mathbf{1 h}$ & $t$ - $\mathrm{Bu}$ & $\mathrm{Cl}$ & $\mathrm{Bn}$ & $p-\mathrm{CH}_{3} \mathrm{C}_{6} \mathrm{H}_{5}$ & 80 \\
$\mathbf{1 i}$ & $t$ - $\mathrm{Bu}$ & $\mathrm{F}$ & $\mathrm{Bn}$ & $p-\mathrm{CH}_{3} \mathrm{C}_{6} \mathrm{H}_{5}$ & 72 \\
$\mathbf{1 j}$ & $t$ - $\mathrm{Bu}$ & $\mathrm{Cl}$ & $\mathrm{Bn}$ & $\mathrm{OCH}_{2} \mathrm{CH}_{3}$ & 75 \\
$\mathbf{1 k}$ & $t$ - $\mathrm{Bu}$ & $\mathrm{CH}$ & $\mathrm{Bn}$ & $\mathrm{OCH}_{2} \mathrm{CH}_{3}$ & 71 \\
$\mathbf{1 l}$ & $t$ - $\mathrm{Bu}$ & $\mathrm{H}$ & $\mathrm{Bn}$ & $\mathrm{OCH}_{3}$ & 69 \\
$\mathbf{1 m}$ & $t$ - $\mathrm{Bu}$ & $\mathrm{Cl}$ & $\mathrm{Bn}$ & $\mathrm{OCH}_{3}$ & 78 \\
\hline
\end{tabular}

${ }^{a}$ Reaction conditions: alkylisocyanide $(0.45 \mathrm{mmol})$, DMAD $(0.45 \mathrm{mmol})$, 3-methyleneoxindole $(0.3 \mathrm{mmol})$, toluene $(10.0 \mathrm{~mL}), 110{ }^{\circ} \mathrm{C}, 0.5 \mathrm{~h} .{ }^{b}$ Isolated yields.

When 3-phencylideneoxindoles with free $\mathrm{NH}$ group were sued in the three-component reaction, the spiro comp- 

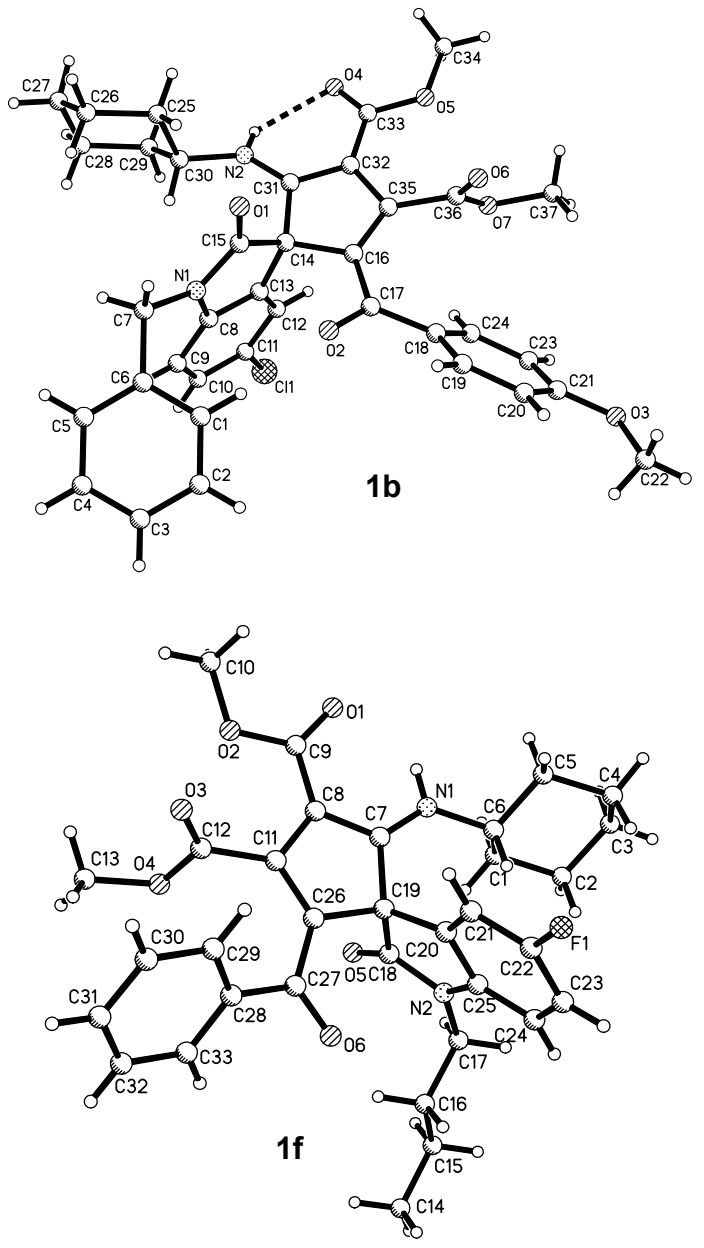

Figure 1 Single crystal structures of the compounds $\mathbf{1 b}$ and $\mathbf{1 f}$

ounds $\mathbf{2 a} \sim \mathbf{2 e}$ were successfully isolated in good yields (Table 3). It can be seen that both the exocyclic $\mathrm{C}=\mathrm{C}$ bond and free $\mathrm{NH}$ group reacted with the in situ generated 1,4-dipole from the reaction of alkylisocyanide with DMAD. The ${ }^{1} \mathrm{H}$ NMR and ${ }^{13} \mathrm{C}$ NMR spectra of the obtained spiro compounds $\mathbf{2 a} \sim \mathbf{2 e}$ clearly showed that they have two units of but-2-enedioate. The nucleophilic addition reaction of primary amine to DMAD usually resulted in the mixture of $Z / E$-isomers of amino-substituted but-2enedioate, which could not be separated due to quick isomerization in solution. The molecular ratios of the $Z / E$-isomers were nearly $1: 9$ according to the ${ }^{1} \mathrm{H}$ NMR spectra. The single crystal structure of the compound 2a was successfully determined by X-ray diffraction (CCDC 2059920, Figure 2). It can be seen that a polysubstituted cyclopentadiene moiety was formed in the molecule. Additionally, the cyclic amino group connected to a 1-aza-buta1,3-diene $(\mathrm{C}=\mathrm{C}-\mathrm{C}=\mathrm{NR})$ scaffold at $\mathrm{C} 2$-position. The two methoxycarbonyl groups exist on the trans-position of the $\mathrm{C}=\mathrm{C}$ bond in the $\mathrm{C}=\mathrm{C}-\mathrm{C}=\mathrm{NR}$ scaffold.

For developing the scope of the reaction, alkyl propiolate was also used in the three-component reaction. The reaction was carried out in refluxing toluene for $3 \mathrm{~h}$. Both methyl and ethyl propiolate reacted smoothly to give the
Table 3 Synthesis of spiro[cyclopentane-1,3'-indolines] 2a $2 \mathrm{e}^{a}$

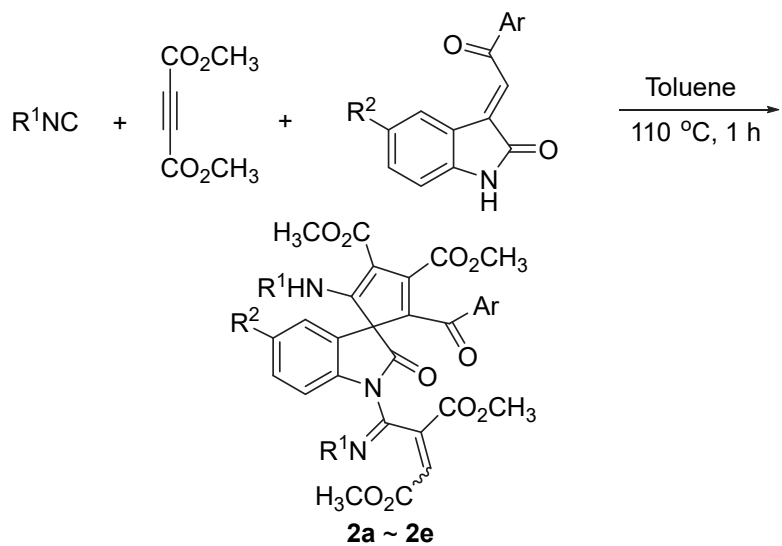

\begin{tabular}{|c|c|c|c|c|}
\hline Compd. & $\mathrm{R}^{1}$ & $\mathrm{R}^{2}$ & $\mathrm{Ar}$ & $\begin{array}{c}\text { Yield }^{b} / \% \\
(Z: E)\end{array}$ \\
\hline $2 a$ & Cyclohexyl & $\mathrm{CH}_{3}$ & $p-\mathrm{CH}_{3} \mathrm{OC}_{6} \mathrm{H}_{5}$ & $63(1: 9)$ \\
\hline $2 \mathrm{~b}$ & Cyclohexyl & $\mathrm{H}$ & $p-\mathrm{CH}_{3} \mathrm{C}_{6} \mathrm{H}_{4}$ & $59(1: 9)$ \\
\hline $2 c$ & $t-\mathrm{Bu}$ & $\mathrm{Cl}$ & $p-\mathrm{CH}_{3} \mathrm{C}_{6} \mathrm{H}_{4}$ & $70(1: 9)$ \\
\hline 2d & $t-\mathrm{Bu}$ & $\mathrm{CH}_{3}$ & $p-\mathrm{ClC}_{6} \mathrm{H}_{4}$ & $64(1: 9)$ \\
\hline $2 e$ & $t-\mathrm{Bu}$ & $\mathrm{F}$ & $p-\mathrm{CH}_{3} \mathrm{OC}_{6} \mathrm{H}_{5}$ & $67(1: 9)$ \\
\hline
\end{tabular}

${ }^{a}$ Reaction conditions: alkylisocyanide $(0.90 \mathrm{mmol})$, DMAD $(0.90 \mathrm{mmol})$, 3-methyleneoxindole $(0.3 \mathrm{mmol})$, toluene $(10.0 \mathrm{~mL}), 110{ }^{\circ} \mathrm{C}, 1.0 \mathrm{~h} .{ }^{b}$ Isolated yields.

expected spiro[cyclopentane-1,3'-indolines] $\mathbf{3 a} \sim \mathbf{3 h}$ in satisfactory yields (Table 4). The results showed that reactivity of alkyl propiolate is relative lower than that of the dialkyl acetylenediacarboxylate. The structures of the spiro compounds were fully characterized by various spectroscopy. The single crystal structure of compound 3a was also successfully determined (CCDC 2059921, Figure 2), which has similar structural character to those of the single crystals $\mathbf{1 b}$ and $\mathbf{1 f}$.

Table 4 Synthesis of spiro[cyclopentane-1,3'-indolines] 3a $3 \mathbf{h}^{a}$

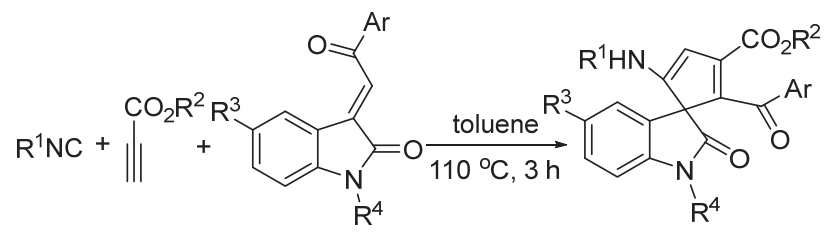

\begin{tabular}{|c|c|c|c|c|c|c|}
\hline Compd. & $\mathrm{R}^{1}$ & $\mathrm{R}^{2}$ & $\mathrm{R}^{3}$ & $\mathrm{R}^{4}$ & $\mathrm{Ar}$ & Yield $b / \%$ \\
\hline $3 \mathbf{a}$ & $t-\mathrm{Bu}$ & $\mathrm{Me}$ & $\mathrm{F}$ & $\mathrm{Bn}$ & $p-\mathrm{CH}_{3} \mathrm{OC}_{6} \mathrm{H}_{5}$ & 88 \\
\hline $3 b$ & $t-\mathrm{Bu}$ & $\mathrm{Me}$ & $\mathrm{F}$ & $\mathrm{Bn}$ & $p-\mathrm{CH}_{3} \mathrm{C}_{6} \mathrm{H}_{5}$ & 83 \\
\hline $3 c$ & $t-\mathrm{Bu}$ & $\mathrm{Me}$ & $\mathrm{H}$ & $\mathrm{Bn}$ & $p-\mathrm{CH}_{3} \mathrm{OC}_{6} \mathrm{H}_{5}$ & 76 \\
\hline 3d & $t-\mathrm{Bu}$ & $\mathrm{Me}$ & $\mathrm{H}$ & $\mathrm{Bn}$ & $p-\mathrm{CH}_{3} \mathrm{C}_{6} \mathrm{H}_{5}$ & 72 \\
\hline $3 e$ & $t-\mathrm{Bu}$ & $\mathrm{Me}$ & $\mathrm{Cl}$ & $\mathrm{Bn}$ & $p-\mathrm{ClC}_{6} \mathrm{H}_{5}$ & 82 \\
\hline $3 f$ & $t-\mathrm{Bu}$ & $\mathrm{Me}$ & $\mathrm{F}$ & $n$-Bu & $\mathrm{C}_{6} \mathrm{H}_{5}$ & 65 \\
\hline $3 g$ & $t-\mathrm{Bu}$ & Et & $\mathrm{Cl}$ & $\mathrm{Bn}$ & $p-\mathrm{CH}_{3} \mathrm{OC}_{6} \mathrm{H}_{5}$ & 72 \\
\hline $3 \mathrm{~h}$ & Cyclohexyl & Et & $\mathrm{Cl}$ & $\mathrm{Bn}$ & $p-\mathrm{ClC}_{6} \mathrm{H}_{5}$ & 76 \\
\hline
\end{tabular}

${ }^{a}$ Reaction conditions: alkylisocyanide $(0.45 \mathrm{mmol})$, alkyl propiolate $(0.45$ mmol), 3-methyleneoxindole $(0.3 \mathrm{mmol})$, toluene $(10.0 \mathrm{~mL}), 110{ }^{\circ} \mathrm{C}, 3.0 \mathrm{~h}$. ${ }^{b}$ Isolated yields. 

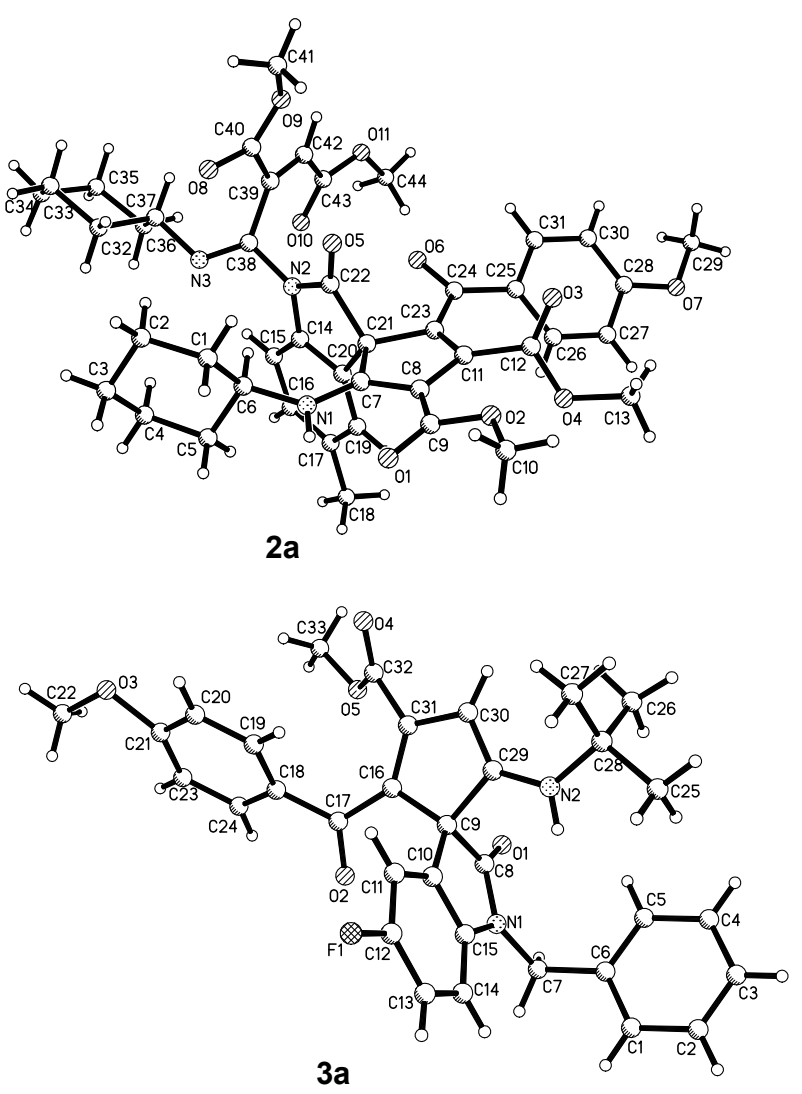

Figure 2 Single crystal structures of the compounds 2a and 3a

In order to demonstrate the synthetic values of this three-component reaction, another kind of common 1,3dipolarophile, 3-arylideneindolin-2-one, was also employed in the reaction. The expected exocyclic $\mathrm{C}=\mathrm{C}$ bond in the substrate could not take part in the reaction at the refluxing toluene for longer time or by employing larger excess of alkylisocyanide and DMAD. However, the NH unit of 3-arylideneindolin-2-ones anticipated in the reaction to give 2-(2-oxoindolin-1-yl)-3-((alkylimino)methylene)succinates $\mathbf{4 a} \sim \mathbf{4 e}$ in high yields (Table 5). This result clearly showed that the exocyclic $\mathrm{C}=\mathrm{C}$ bonds in 3-arylideneindolin-2-ones are not active in the reaction due to lacking of the carbonyl group comparing to the 3-pheacylideneoxindoles. The single crystal structure of the compound 4a was determined by X-ray diffraction method (CCDC 2059922). From Figure 5, it can be seen that the exocyclic $\mathrm{C}=\mathrm{C}$ bond has a $Z$-configuration, in which the phenyl group and the carbonyl group in oxindolin-2-one exist on cis-position. It should be pointed out that 3 -arylideneoxindolin-2-ones were easy to be prepared as an $E / Z$ mixture with high ratio. ${ }^{[17]}$ Here, a single crystal with $Z$-configuration was easily formed. The cyclic amino group connected to a 1-aza-buta-1,2-diene $(\mathrm{C}-\mathrm{C}=\mathrm{C}=$ $\mathrm{NR}$ ) scaffold at $\mathrm{C} 4$-position in the molecule 4a. Comparing with the above single crystal structure $\mathbf{2 a}$ in Figure 2, in which the amino group connected to an isomeric 1-azabuta-1,3-diene $(\mathrm{C}=\mathrm{C}-\mathrm{C}=\mathrm{NR})$ scaffold at $\mathrm{C} 2$-position in the molecule. A literature survey indicated that the for- mation of 1-aza-buta-1,3-diene $(\mathrm{C}=\mathrm{C}-\mathrm{C}=\mathrm{NR})$ scaffold was often observed in the reaction of the domino reaction of isocyanide and electron-deficient alkynes, ${ }^{[9-10]}$ while the formation of 1-aza-buta-1,2-diene $(\mathrm{C}-\mathrm{C}=\mathrm{C}=\mathrm{NR})$ scaffold was occasionally encountered. ${ }^{[18]}$

Table 5 Synthesis of functionalized indolin-2-ones $\mathbf{4 a} \sim \mathbf{4} \mathrm{e}^{a}$

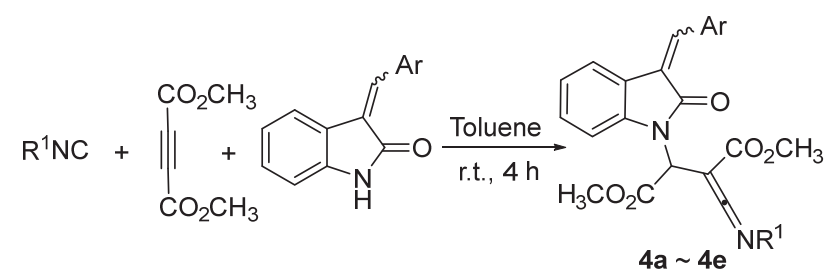

\begin{tabular}{cllc}
\hline Compd. & \multicolumn{1}{c}{$\mathrm{R}^{1}$} & \multicolumn{1}{c}{$\mathrm{Ar}$} & $\mathrm{Yield}^{b} / \%$ \\
\hline $\mathbf{4 a}$ & $t-\mathrm{Bu}$ & $p-\mathrm{CH}_{3} \mathrm{OC}_{6} \mathrm{H}_{4}$ & 74 \\
$\mathbf{4 b}$ & $t-\mathrm{Bu}$ & $p-\mathrm{ClC}_{6} \mathrm{H}_{4}$ & 79 \\
$\mathbf{4 c}$ & $t-\mathrm{Bu}$ & $p-\mathrm{BrC}_{6} \mathrm{H}_{4}$ & 75 \\
$\mathbf{4 d}$ & $t-\mathrm{Bu}$ & $m-\mathrm{ClC}_{6} \mathrm{H}_{4}$ & 68 \\
$\mathbf{4 e}$ & $\mathrm{Cyclohexyl}$ & $p-\mathrm{ClC}_{6} \mathrm{H}_{4}$ & 77 \\
\hline
\end{tabular}

${ }^{a}$ Reaction conditions: alkylisocyanide $(0.30 \mathrm{mmol})$, DMAD $(0.30 \mathrm{mmol})$, 3-arylideneindolin-2-one $(0.3 \mathrm{mmol})$, toluene $(10.0 \mathrm{~mL})$, r.t., 4.0 h. ${ }^{b}$ Isolated yields.

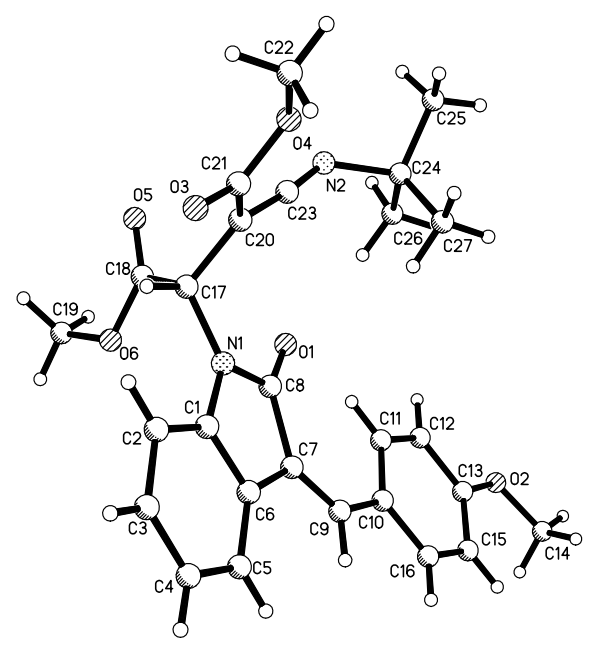

Figure 5 Single crystal structure of the compound $4 \mathbf{a}$

In order to explain the formation of different spiro compounds, a plausible reaction mechanism is proposed in Scheme 1 on the basis of the previous reported works ${ }^{[9-10]}$ and the present experiments. At first, a nucleophilic addition of alkylisocyanide to DMAD results in a 1,4-dipolar intermediate A. Secondly, Michael addition of the 1,4dipolar intermediate A to 3-phenacylideneoxindole gives the adduct $\mathbf{B}$. Thirdly, the intramolecular coupling of the carbanion to the ammonium affords an imino-substituted intermediate $\mathbf{C}$. Then, the isolated spiro compound $\mathbf{1}$ or $\mathbf{3}$ is formed by $1,5-\mathrm{H}$ shift process. In the case of 3-phenacylideneoxindole with a free $\mathrm{NH}$ group $\left(\mathrm{R}^{3}=\mathrm{H}\right)$, the further reaction of free $\mathrm{NH}$ group with excess 1,4-dipolar intermediate $\mathbf{A}$ give the spiro compound 2 . When 3-arylideneoxindolin-2-one is employed in the reaction, the exocyclic $\mathrm{C}=\mathrm{C}$ bond is not active enough to react with the 
1,4-dipolar intermediate A. Alternatively, the protonation of intermediate $\mathbf{A}$ gives the intermediate $\mathbf{D}$, while 3-arylideneoxindolin-2-one converts to an amino anion $\mathbf{E}$. Then, Michael addition of the anion $\mathbf{E}$ to the intermediate $\mathbf{D}$ results in the adduct $\mathbf{F}$, which in turn isomerizes to give the isolated product 4 with a 1-aza-buta-1,2-diene $(\mathrm{C}-\mathrm{C}=$ $\mathrm{C}=\mathrm{NR}$ ) scaffold.

\section{Conclusions}

In summary, we have investigated the three-component reaction of alkylisocyanide, dialkyl acetylenedicarboxylate (alkyl propiolate) and 3-phenacylideneoxindoles. This reaction successfully provided an efficient synthetic protocol for the functionalized spiro[cyclopentane-1,3'-indolines] in satisfactory yields. It is interesting to find that the free $\mathrm{NH}$ group in 3-phenacylideneoxindoles and 3-arylideneoxindolin-2-ones could react with alkylisocyanide and dialkyl acetylenedicarboxylate to give the scaffolds containing 1aza-buta-1,3-diene or 1-aza-buta-1,2-diene unit. The stereochemistry of the various spiro compounds was confirmed by determination of several single crystal structures. This reaction not only provided efficient synthetic methodology for biologically important spirooxindoles, but also developed the high efficiency and atom-economy of the multicomponent reaction. The potential applications of this multicomponent reaction in organic synthesis and medicinal chemistry might be significant.

\section{Experimental section}

4.1 General procedure for the synthesis of spiro[cyclopentane-1,3'-indolines] (1a $\sim \mathbf{1 m})$

A mixture of alkylisocyanide $(0.45 \mathrm{mmol})$, DMAD $(0.45 \mathrm{mmol})$ and 3 -methyleneoxindole $(0.3 \mathrm{mmol})$ in toluene $(10.0 \mathrm{~mL})$ was refluxed at $110{ }^{\circ} \mathrm{C}$ for $0.5 \mathrm{~h}$. Then, the solvent was removed by rotatory evaporation at reduced pressure. The residue was subjected to column chromatography with petroleum ether and ethyl acetate $(V: V=4$ : 1) as eluent to give the pure product.

Dimethyl 1'-benzyl-2-(cyclohexylamino)-5-(4-methylbenzoyl)-2'-oxospiro[cyclopentane-1,3'-indoline]-2,4diene-3,4-dicarboxylate (1a): Yellow solid, 82\% yield. m.p. $235 \sim 237{ }^{\circ} \mathrm{C} ;{ }^{1} \mathrm{H}$ NMR $\left(400 \mathrm{MHz}, \mathrm{CDCl}_{3}\right) \delta: 8.91(\mathrm{~d}$, $J=9.6 \mathrm{~Hz}, 1 \mathrm{H}, \mathrm{NH}), 7.60$ (d, $J=7.2 \mathrm{~Hz}, 2 \mathrm{H}, \operatorname{ArH}), 7.39 \sim$ 7.35 (m, 4H, ArH), 7.30 (d, J=7.2 Hz, 1H, ArH), $7.21(\mathrm{t}$, $J=8.0 \mathrm{~Hz}, 1 \mathrm{H}, \mathrm{ArH}), 7.15 \sim 7.10(\mathrm{~m}, 3 \mathrm{H}, \mathrm{ArH}), 6.98(\mathrm{t}$, $J=7.4 \mathrm{~Hz}, 1 \mathrm{H}, \mathrm{ArH}), 6.84(\mathrm{~d}, J=7.6 \mathrm{~Hz}, 1 \mathrm{H}, \mathrm{ArH}), 5.05$ (s, $\left.2 \mathrm{H}, \mathrm{CH}_{2}\right), 3.71\left(\mathrm{~s}, 3 \mathrm{H}, \mathrm{OCH}_{3}\right), 3.22\left(\mathrm{~s}, 3 \mathrm{H}, \mathrm{OCH}_{3}\right), 2.75(\mathrm{~d}$, $J=10.0 \mathrm{~Hz}, 1 \mathrm{H}, \mathrm{CH}), 2.33\left(\mathrm{~s}, 3 \mathrm{H}, \mathrm{CH}_{3}\right), 1.71$ (d, $J=9.6$ $\left.\mathrm{Hz}, 1 \mathrm{H}, \mathrm{CH}_{2}\right), 1.35 \sim 1.26\left(\mathrm{~m}, 3 \mathrm{H}, \mathrm{CH}_{2}\right), 1.17 \sim 1.14(\mathrm{~m}$, $\left.1 \mathrm{H}, \mathrm{CH}_{2}\right), 1.01 \sim 0.92\left(\mathrm{~m}, 2 \mathrm{H}, \mathrm{CH}_{2}\right), 0.88 \sim 0.78(\mathrm{~m}, 2 \mathrm{H}$, $\left.\mathrm{CH}_{2}\right), 0.55$ (d, $\left.J=10.0 \mathrm{~Hz}, 1 \mathrm{H}, \mathrm{CH}_{2}\right) ;{ }^{13} \mathrm{C} \mathrm{NMR}(100 \mathrm{MHz}$, $\left.\mathrm{CDCl}_{3}\right) \delta: 188.9,171.6,170.9,165.7,165.1,144.6,141.4$, $136.8,135.7,129.6,128.9,128.3,128.3,128.1,127.8$, $126.0,123.1,123.1,110.0,64.9,53.9,51.6,51.1,45.7$,

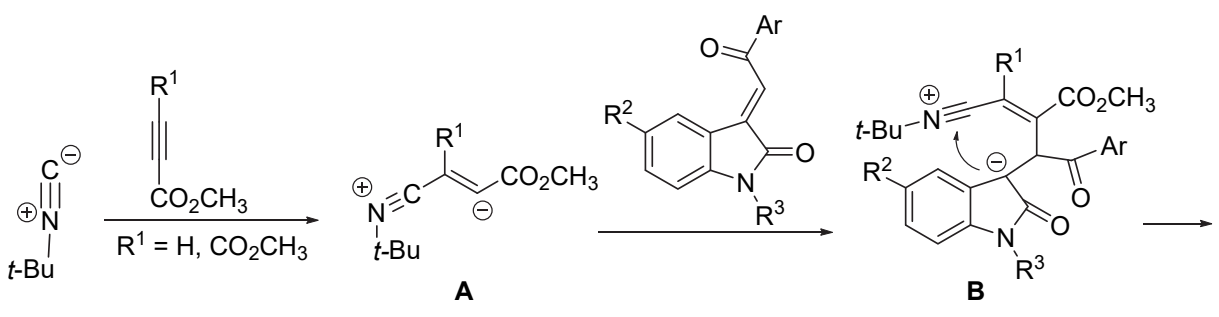

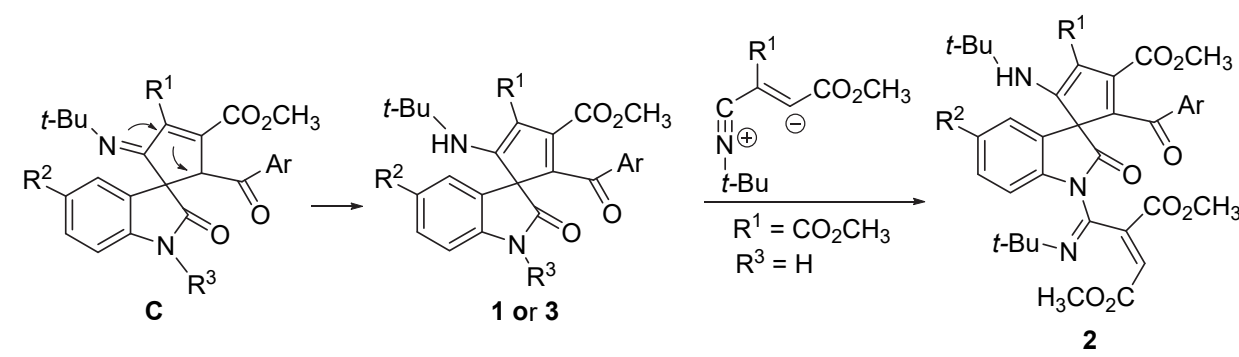<smiles>COC(=O)C(C#N)=CC(=O)OCC(C)(C)C</smiles><smiles>O=C1Nc2ccccc2/C1=C/Br</smiles><smiles>C1CCCCC1</smiles>

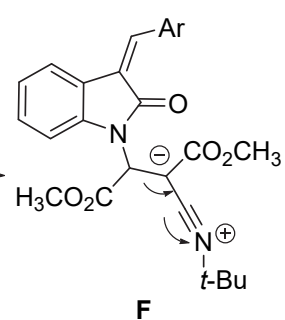<smiles>COC(=O)C(C(=NC(C)(C)C)OC(C)=O)N1C(=O)C(=CBr)c2ccccc21</smiles>

Scheme 1 Plausible reaction mechanism for various products $1 \sim 4$ 
33.9, 33.6, 24.8, 24.3, 24.2, 21.5; IR (KBr) v: 3244, 2935, 2855, 1753, 1717, 1665, 1602, 1551, 1459, 1336, 1213, 1082, 1039, 964, 799, 754, 707, 589, $549 \mathrm{~cm}^{-1}$; HRMS (ESI) calcd for $\mathrm{C}_{37} \mathrm{H}_{37} \mathrm{~N}_{2} \mathrm{O}_{6}[\mathrm{M}+\mathrm{H}]^{+}$605.2652, found 605.2648 .

Dimethyl 1'-benzyl-5'-chloro-2-(cyclohexylamino)-5-(4methoxybenzoyl)-2'-oxospiro[cyclopentane-1,3'-indoline]2,4-diene-3,4-dicarboxylate (1b): Yellow solid, 86\% yield. m.p. 199 $201{ }^{\circ} \mathrm{C} ;{ }^{1} \mathrm{H}$ NMR (400 MHz, $\left.\mathrm{CDCl}_{3}\right) \delta: 8.93(\mathrm{~d}$, $J=10.4 \mathrm{~Hz}, 1 \mathrm{H}, \mathrm{NH}), 7.57$ (d, $J=7.2 \mathrm{~Hz}, 2 \mathrm{H}, \mathrm{ArH}), 7.49$ (d, $J=8.8 \mathrm{~Hz}, 2 \mathrm{H}, \mathrm{ArH}), 7.37$ (t, $J=7.6 \mathrm{~Hz}, 2 \mathrm{H}, \mathrm{ArH}$ ), 7.30 (t, $J=7.2 \mathrm{~Hz}, 1 \mathrm{H}, \mathrm{ArH}), 7.20 \sim 7.17$ (m, 1H, ArH), $7.13(\mathrm{~d}, J=1.6 \mathrm{~Hz}, 1 \mathrm{H}, \operatorname{ArH}), 6.83(\mathrm{~d}, J=8.8 \mathrm{~Hz}, 2 \mathrm{H}$, $\operatorname{ArH}), 6.76(\mathrm{~d}, J=8.4 \mathrm{~Hz}, 1 \mathrm{H}, \mathrm{ArH}), 5.08 \sim 4.99(\mathrm{~m}, 2 \mathrm{H}$, $\left.\mathrm{CH}_{2}\right), 3.81\left(\mathrm{~s}, 3 \mathrm{H}, \mathrm{OCH}_{3}\right), 3.72\left(\mathrm{~s}, 3 \mathrm{H}, \mathrm{OCH}_{3}\right), 3.30(\mathrm{~s}, 3 \mathrm{H}$, $\left.\mathrm{OCH}_{3}\right), 2.73 \sim 2.70(\mathrm{~m}, 1 \mathrm{H}, \mathrm{CH}), 1.72,(\mathrm{~d}, J=9.2 \mathrm{~Hz}, 1 \mathrm{H}$, $\left.\mathrm{CH}_{2}\right), 1.45\left(\mathrm{~d}, J=15.6 \mathrm{~Hz}, 1 \mathrm{H}, \mathrm{CH}_{2}\right), 1.36(\mathrm{~d}, J=13.2 \mathrm{~Hz}$, $\left.1 \mathrm{H}, \mathrm{CH}_{2}\right), 1.26 \sim 1.15\left(\mathrm{~m}, 2 \mathrm{H}, \mathrm{CH}_{2}\right), 1.02(\mathrm{~d}, J=4.8 \mathrm{~Hz}$, $\left.2 \mathrm{H}, \mathrm{CH}_{2}\right), 0.81 \sim 0.60\left(\mathrm{~m}, 2 \mathrm{H}, \mathrm{CH}_{2}\right), 0.62(\mathrm{~d}, J=13.6 \mathrm{~Hz}$, $\left.1 \mathrm{H}, \mathrm{CH}_{2}\right) ;{ }^{13} \mathrm{C} \mathrm{NMR}\left(100 \mathrm{MHz}, \mathrm{CDCl}_{3}\right) \delta: 188.1,171.3$, $170.1,165.6,164.9,162.2,149.6,143.2,135.2,131.9$, $130.4,129.5,128.9,128.5,127.9,127.9,127.7,126.6$, 123.3, 112.9, 110.9, 99.4, 64.7, 55.3, 54.1, 51.8, 51.2, 45.8, $33.9,33.6,24.7,24.6,24.2$; IR (KBr) v: 2939, 2848, 1733, 1666, 1597, 1552, 1457, 1328, 1251, 1216, 1170, 1035, $798,743,699,599 \mathrm{~cm}^{-1}$; HRMS (ESI) calcd for $\mathrm{C}_{37} \mathrm{H}_{36} \mathrm{Cl}-$ $\mathrm{N}_{2} \mathrm{O}_{7}[\mathrm{M}+\mathrm{H}]^{+}$655.2211, found 655.2199.

Dimethyl 1'-benzyl-5'-chloro-2-(cyclohexylamino)-5-(4methylbenzoyl)-2'-oxospiro[cyclopentane-1,3'-indoline]2,4-diene-3,4-dicarboxylate (1c): Yellow solid, 82\% yield. m.p. $219 \sim 221{ }^{\circ} \mathrm{C} ;{ }^{1} \mathrm{H}$ NMR $\left(400 \mathrm{MHz}, \mathrm{CDCl}_{3}\right) \delta: 8.96(\mathrm{~d}$, $J=10.4 \mathrm{~Hz}, 1 \mathrm{H}, \mathrm{NH}), 7.57$ (d, $J=7.2 \mathrm{~Hz}, 2 \mathrm{H}, \mathrm{ArH})$, $7.39 \sim 7.35(\mathrm{~m}, 4 \mathrm{H}, \mathrm{ArH}), 7.32 \sim 7.29(\mathrm{~m}, 1 \mathrm{H}, \mathrm{ArH})$, $7.20 \sim 7.18(\mathrm{~m}, 1 \mathrm{H}, \mathrm{ArH}), 7.13 \sim 7.12(\mathrm{~m}, 1 \mathrm{H}, \mathrm{ArH}), 6.76$ $(\mathrm{d}, J=8.0 \mathrm{~Hz}, 1 \mathrm{H}, \mathrm{ArH}), 5.09 \sim 4.98\left(\mathrm{~m}, 2 \mathrm{H}, \mathrm{CH}_{2}\right), 3.72$ $\left(\mathrm{s}, 3 \mathrm{H}, \mathrm{OCH}_{3}\right), 3.23\left(\mathrm{~s}, 3 \mathrm{H}, \mathrm{OCH}_{3}\right), 2.73(\mathrm{~d}, J=9.6 \mathrm{~Hz}$, $1 \mathrm{H}, \mathrm{CH}), 2.35$ (s, 3H, $\left.\mathrm{CH}_{3}\right), 1.72(\mathrm{~d}, J=12.4 \mathrm{~Hz}, 1 \mathrm{H}$, $\left.\mathrm{CH}_{2}\right), 1.45\left(\mathrm{~d}, J=13.2 \mathrm{~Hz}, 1 \mathrm{H}, \mathrm{CH}_{2}\right), 1.36(\mathrm{~d}, J=13.2 \mathrm{~Hz}$, $\left.1 \mathrm{H}, \mathrm{CH}_{2}\right), 1.26 \sim 1.15\left(\mathrm{~m}, 2 \mathrm{H}, \mathrm{CH}_{2}\right), 1.05 \sim 0.99(\mathrm{~m}, 2 \mathrm{H}$, $\left.\mathrm{CH}_{2}\right), 0.92 \sim 0.82\left(\mathrm{~m}, 2 \mathrm{H}, \mathrm{CH}_{2}\right), 0.62(\mathrm{~d}, J=13.2 \mathrm{~Hz}, 1 \mathrm{H}$, $\left.\mathrm{CH}_{2}\right) ;{ }^{13} \mathrm{C} \mathrm{NMR}\left(100 \mathrm{MHz}, \mathrm{CDCl}_{3}\right) \delta: 188.8,171.3,170.3$, $165.6,164.9,143.2,141.5,136.6,135.2,129.5,128.9$, $128.4,128.3,128.2,127.9,127.9,127.8,123.3,110.9$, $99.5,64.6,54.1,51.7,51.2,45.8,33.9,33.5,24.7,24.4$, 24.2, 21.5; IR (KBr) v: 3238, 2927, 2848, 1734, 1663, 1613, 1551, 1457, 1333, 1247, 1208, 1042, 826, 742, 701, $596 \mathrm{~cm}^{-1}$; HRMS (ESI) calcd for $\mathrm{C}_{37} \mathrm{H}_{36} \mathrm{ClN}_{2} \mathrm{O}_{6}[\mathrm{M}+\mathrm{H}]^{+}$ 639.2262, found 639.2250.

Dimethyl 1'-benzyl-2-(cyclohexylamino)-5'-fluoro-5-(4methoxybenzoyl)-2'-oxospiro[cyclopentane-1,3'-indoline]2,4-diene-3,4-dicarboxylate (1d): Yellow solid, 78\% yield. m.p. $190 \sim 192{ }^{\circ} \mathrm{C} ;{ }^{1} \mathrm{H}$ NMR (400 MHz, $\left.\mathrm{CDCl}_{3}\right) \delta: 8.93$ (d, $J=10.0 \mathrm{~Hz}, 1 \mathrm{H}, \mathrm{NH}), 7.58$ (d, $J=7.6 \mathrm{~Hz}, 2 \mathrm{H}, \mathrm{ArH})$, 7.49 (d, $J=8.8 \mathrm{~Hz}, 2 \mathrm{H}, \mathrm{ArH}), 7.37$ (t, $J=6.8 \mathrm{~Hz}, 2 \mathrm{H}$, ArH), $7.32 \sim 7.29(\mathrm{~m}, 1 \mathrm{H}, \mathrm{ArH}), 6.94 \sim 6.90(\mathrm{~m}, 1 \mathrm{H}, \mathrm{ArH})$, $6.82(\mathrm{~d}, J=8.8 \mathrm{~Hz}, 2 \mathrm{H}, \mathrm{ArH}), 6.78 \sim 6.74(\mathrm{~m}, 1 \mathrm{H}, \mathrm{ArH})$, $5.09 \sim 5.00\left(\mathrm{~m}, 2 \mathrm{H}, \mathrm{CH}_{2}\right), 3.81\left(\mathrm{~s}, 3 \mathrm{H}, \mathrm{OCH}_{3}\right), 3.72(\mathrm{~s}, 3 \mathrm{H}$,
$\left.\mathrm{OCH}_{3}\right), 3.29\left(\mathrm{~s}, 3 \mathrm{H}, \mathrm{OCH}_{3}\right), 2.75(\mathrm{~d}, J=10.4 \mathrm{~Hz}, 1 \mathrm{H}, \mathrm{CH})$, $1.73\left(\mathrm{~d}, J=12.4 \mathrm{~Hz}, 1 \mathrm{H}, \mathrm{CH}_{2}\right), 1.46 \sim 1.35\left(\mathrm{~m}, 2 \mathrm{H}, \mathrm{CH}_{2}\right)$, $1.26 \sim 1.15\left(\mathrm{~m}, 2 \mathrm{H}, \mathrm{CH}_{2}\right), 1.05 \sim 0.98(\mathrm{~m}, 2 \mathrm{H}, \mathrm{ArH})$, $0.95 \sim 0.82\left(\mathrm{~m}, 2 \mathrm{H}, \mathrm{CH}_{2}\right), 0.61\left(\mathrm{~d}, J=13.2 \mathrm{~Hz}, 1 \mathrm{H}, \mathrm{CH}_{2}\right)$; ${ }^{13} \mathrm{C} \mathrm{NMR}\left(100 \mathrm{MHz}, \mathrm{CDCl}_{3}\right) \delta: 188.1,171.4,170.1,165.6$, $165.0,162.2,159.1$ (d, $J=241.3 \mathrm{~Hz}), 149.5,140.7,135.3$, $131.9,130.4,128.9,127.9,127.9,127.6,127.5,126.7$, $115.9(\mathrm{~d}, J=23.3 \mathrm{~Hz}), 112.9,111.1(\mathrm{~d}, J=25.1 \mathrm{~Hz}), 110.6$ (d, $J=8.0 \mathrm{~Hz}), 99.41,65.06,55.35,54.01,51.83,51.20$, 45.92, 34.00, 33.58, 24.73, 24.36, 24.18; IR (KBr) v: 3235, 3059, 2939, 2854, 1722, 1672, 1601, 1551, 1456, 1336, 1219, 1167, 1081, 1015, 927, 888, 792, 703, 606, 538 $\mathrm{cm}^{-1}$; HRMS (ESI) calcd for $\mathrm{C}_{37} \mathrm{H}_{36} \mathrm{FN}_{2} \mathrm{O}_{7}[\mathrm{M}+\mathrm{H}]^{+}$ 639.2507, found 639.2488.

Dimethyl 1'-benzyl-2-(cyclohexylamino)-5'-fluoro-5(4-methylbenzoyl)-2'-oxospiro[cyclopentane-1,3'-indoline]-2,4-diene-3,4-dicarboxylate (1e): Yellow solid, 75\% yield. m.p. $185 \sim 187{ }^{\circ} \mathrm{C} ;{ }^{1} \mathrm{H}$ NMR $\left(400 \mathrm{MHz}, \mathrm{CDCl}_{3}\right) \delta$ : $8.95(\mathrm{~d}, J=10.8 \mathrm{~Hz}, 1 \mathrm{H}, \mathrm{NH}), 7.58(\mathrm{~d}, J=7.6 \mathrm{~Hz}, 2 \mathrm{H}$, ArH), $7.39 \sim 7.37(\mathrm{~m}, 2 \mathrm{H}, \mathrm{ArH}), 7.32 \sim 7.27(\mathrm{~m}, 2 \mathrm{H}, \mathrm{ArH})$, $7.13(\mathrm{~d}, J=7.2 \mathrm{~Hz} 2 \mathrm{H}, \mathrm{ArH}), 6.94 \sim 6.90(\mathrm{~m}, 2 \mathrm{H}, \mathrm{ArH})$, $6.77 \sim 6.74(\mathrm{~m}, 1 \mathrm{H}, \mathrm{ArH}), 5.09 \sim 4.99\left(\mathrm{~m}, 2 \mathrm{H}, \mathrm{CH}_{2}\right), 3.72$ $\left(\mathrm{s}, 3 \mathrm{H}, \mathrm{OCH}_{3}\right), 3.23\left(\mathrm{~s}, 3 \mathrm{H}, \mathrm{OCH}_{3}\right), 2.76(\mathrm{~d}, J=10.0 \mathrm{~Hz}$, $1 \mathrm{H}, \mathrm{CH}), 2.34\left(\mathrm{~s}, 3 \mathrm{H}, \mathrm{CH}_{3}\right), 1.73$ (d, $J=13.2 \mathrm{~Hz}, 1 \mathrm{H}$, $\left.\mathrm{CH}_{2}\right), 1.46 \sim 1.35\left(\mathrm{~m}, 2 \mathrm{H}, \mathrm{CH}_{2}\right), 1.27 \sim 1.18\left(\mathrm{~m}, 2 \mathrm{H}, \mathrm{CH}_{2}\right)$, $1.05 \sim 1.01\left(\mathrm{~m}, 2 \mathrm{H}, \mathrm{CH}_{2}\right), 0.96 \sim 0.83\left(\mathrm{~m}, 2 \mathrm{H}, \mathrm{CH}_{2}\right), 0.61$ $\left(\mathrm{d}, J=12.0 \mathrm{~Hz}, 1 \mathrm{H}, \mathrm{CH}_{2}\right) ;{ }^{13} \mathrm{C} \mathrm{NMR}\left(100 \mathrm{MHz} \mathrm{CDCl}_{3}\right) \delta$ : $188.9,171.4,170.3,165.6,164.9,159.2$ (d, $J=241.5 \mathrm{~Hz})$, $150.2,141.5,140.8,136.6,135.3,128.9,128.3,128.2$, $127.9,127.9,127.7,127.6,126.7,115.9(\mathrm{~d}, J=23.1 \mathrm{~Hz})$, $111.1(\mathrm{~d}, J=25.1 \mathrm{~Hz}), 110.6(\mathrm{~d}, J=7.7 \mathrm{~Hz}), 99.5,64.9$, 54.1, 51.7, 51.2, 45.9, 33.9, 33.6, 24.7, 24.4, 24.2, 21.5; IR (KBr) v: 3232, 2936, 2857, 1728, 1664, 1600, 1542, 1491, 1457, 1341, 1250, 1216, 1157, 1083, 1040, 825, 791, 751, $705,597 \mathrm{~cm}^{-1}$; HRMS (ESI) calcd for $\mathrm{C}_{37} \mathrm{H}_{36} \mathrm{FN}_{2} \mathrm{O}_{6}[\mathrm{M}+$ $\mathrm{H}]^{+}$623.2557, found 623.2547 .

Dimethyl 2-benzoyl-1'-butyl-5-(cyclohexylamino)-5'fluoro-2'-oxospiro[cyclopentane-1,3'-indoline]-2,4-diene3,4-dicarboxylate (1f): Yellow solid, 62\% yield. m.p. $163 \sim 165{ }^{\circ} \mathrm{C} ;{ }^{1} \mathrm{H}$ NMR (400 MHz, $\left.\mathrm{CDCl}_{3}\right) \delta: 8.92(\mathrm{~d}, J=$ $8.8 \mathrm{~Hz}, 1 \mathrm{H}, \mathrm{NH}), 7.43 \sim 7.37(\mathrm{~m}, 3 \mathrm{H}, \mathrm{ArH}), 7.32 \sim 7.28$ $(\mathrm{m}, 2 \mathrm{H}, \mathrm{ArH}), 7.05 \sim 7.01(\mathrm{~m}, 1 \mathrm{H}, \mathrm{ArH}), 6.93 \sim 6.86(\mathrm{~m}$, $2 \mathrm{H}, \mathrm{ArH}), 3.91 \sim 3.75\left(\mathrm{~m}, 2 \mathrm{H}, \mathrm{CH}_{2}\right), 3.71\left(\mathrm{~s}, 3 \mathrm{H}, \mathrm{OCH}_{3}\right)$, $3.19\left(\mathrm{~s}, 3 \mathrm{H}, \mathrm{OCH}_{3}\right), 2.80(\mathrm{~d}, J=10.4 \mathrm{~Hz}, 1 \mathrm{H}, \mathrm{CH}), 1.86 \sim$ $1.80\left(\mathrm{~m}, 3 \mathrm{H}, \mathrm{CH}_{2}\right), 1.70\left(\mathrm{~s}, 1 \mathrm{H}, \mathrm{CH}_{2}\right), 1.57 \sim 1.46(\mathrm{~m}, 5 \mathrm{H}$, $\left.\mathrm{CH}_{2}\right), 1.26 \sim 1.20\left(\mathrm{~m}, 1 \mathrm{H}, \mathrm{CH}_{2}\right), 1.07\left(\mathrm{~s}, 1 \mathrm{H}, \mathrm{CH}_{2}\right), 1.02(\mathrm{t}$, $\left.J=7.2 \mathrm{~Hz}, 3 \mathrm{H}, \mathrm{CH}_{3}, \mathrm{CH}_{2}\right), 0.72\left(\mathrm{~d}, J=8.4 \mathrm{~Hz}, 1 \mathrm{H}, \mathrm{CH}_{2}\right)$; ${ }^{13} \mathrm{C} \mathrm{NMR}\left(100 \mathrm{MHz}, \mathrm{CDCl}_{3}\right) \delta: 188.9,170.8,170.6,165.6$, $164.8,159.0$ (d, $J=241.1 \mathrm{~Hz}), 150.7,140.9,139.3,130.8$, $127.9,127.7,127.6,126.3,115.9$ (d, $J=23.2 \mathrm{~Hz}), 111.1$ $(\mathrm{d}, J=24.9 \mathrm{~Hz}), 109.6(\mathrm{~d}, J=8.0 \mathrm{~Hz}), 99.5,64.8,54.1$, 51.6, 51.2, 41.2, 33.9, 33.7, 29.2, 24.8, 24.7, 24.5, 20.4, 13.7; IR (KBr) v: 3245, 3062, 2934, 2858, 1730, 1671, $1604,1547,1485,1454,1339,1222,1154,1086,1048$, 997, 927, 891, 812, 781, 714, 656, 581, $549 \mathrm{~cm}^{-1}$; HRMS (ESI) calcd for $\mathrm{C}_{33} \mathrm{H}_{36} \mathrm{FN}_{2} \mathrm{O}_{6}[\mathrm{M}+\mathrm{H}]^{+}$575.2557, found 575.2560 . 
Dimethyl 1'-benzyl-2-(tert-butylamino)-5'-chloro-5-(4methoxybenzoyl)-2'-oxospiro[cyclopentane-1,3'-indoline]2,4-diene-3,4-dicarboxylate (1g): Yellow solid, 85\% yield. m.p. $184 \sim 186{ }^{\circ} \mathrm{C} ;{ }^{1} \mathrm{H}$ NMR (400 MHz, $\left.\mathrm{CDCl}_{3}\right) \delta: 10.00$ (s, 1H, NH), $7.54(\mathrm{~d}, J=7.2 \mathrm{~Hz}, 2 \mathrm{H}, \operatorname{ArH}), 7.46(\mathrm{~d}, J=8.4$ $\mathrm{Hz}, 2 \mathrm{H}, \mathrm{ArH}), 7.36 \sim 7.29(\mathrm{~m}, 3 \mathrm{H}, \mathrm{ArH}), 7.18 \sim 7.15(\mathrm{~m}$, 1H, ArH), 7.09 (d, $J=2.0 \mathrm{~Hz}, 1 \mathrm{H}, \operatorname{ArH}), 6.81$ (d, $J=8.4$ $\mathrm{Hz}, 2 \mathrm{H}, \mathrm{ArH}), 6.75$ (d, J=8.4 Hz, 1H, ArH), 5.01 (s, 2H, $\left.\mathrm{CH}_{2}\right), 3.81\left(\mathrm{~s}, 3 \mathrm{H}, \mathrm{OCH}_{3}\right), 3.72\left(\mathrm{~s}, 3 \mathrm{H}, \mathrm{OCH}_{3}\right), 3.08(\mathrm{~s}, 3 \mathrm{H}$, $\left.\mathrm{OCH}_{3}\right), 1.01\left(\mathrm{~s}, 9 \mathrm{H}, \mathrm{CH}_{3}\right) ;{ }^{13} \mathrm{C} \mathrm{NMR}\left(100 \mathrm{MHz}, \mathrm{CDCl}_{3}\right) \delta$ : $188.2,171.7,170.4,166.1,165.1,162.3,147.7,143.6$, $134.8,132.1,130.6,129.6,128.7,128.4,128.3,128.3$, $127.9,127.9,123.6,112.9,111.2,101.7,65.4,55.6,55.4$, 51.8, 51.2, 46.3, 30.6; IR (KBr) v: 2948, 1731, 1663, 1563, 1454, 1411, 1370, 1322, 1255, 1214, 1031, 916, 820, 781, $743,697,600,548 \mathrm{~cm}^{-1}$; HRMS (ESI) calcd for $\mathrm{C}_{35} \mathrm{H}_{33} \mathrm{Cl}-$ $\mathrm{N}_{2} \mathrm{NaO}_{7}[\mathrm{M}+\mathrm{H}]^{+}$651.1874, found 651.1872.

Dimethyl 1'-benzyl-2-(tert-butylamino)-5'-chloro-5-(4methylbenzoyl)-2'-oxospiro[cyclopentane-1,3'-indoline]2,4-diene-3,4-dicarboxylate $(\mathbf{1 h})$ : Yellow solid, 80\% yield. m.p. 201 203 ${ }^{\circ} \mathrm{C}$; ${ }^{1} \mathrm{H}$ NMR (400 MHz, $\left.\mathrm{CDCl}_{3}\right) \delta: 10.04$ $(\mathrm{s}, 1 \mathrm{H}, \mathrm{NH}), 7.55(\mathrm{~d}, J=7.2 \mathrm{~Hz}, 2 \mathrm{H}, \mathrm{ArH}), 7.36 \sim 7.29(\mathrm{~m}$, $5 \mathrm{H}, \mathrm{ArH}), 7.18 \sim 7.16(\mathrm{~m}, 1 \mathrm{H}, \mathrm{ArH}), 7.13 \sim 7.09(\mathrm{~m}, 3 \mathrm{H}$, $\operatorname{ArH}), 6.75(\mathrm{~d}, J=8.4 \mathrm{~Hz}, 1 \mathrm{H}, \mathrm{ArH}), 5.01\left(\mathrm{~s}, 2 \mathrm{H}, \mathrm{CH}_{2}\right)$, $3.71\left(\mathrm{~s}, 3 \mathrm{H}, \mathrm{OCH}_{3}\right), 3.19\left(\mathrm{~s}, 3 \mathrm{H}, \mathrm{OCH}_{3}\right), 2.34\left(\mathrm{~s}, 3 \mathrm{H}, \mathrm{CH}_{3}\right)$, 1.01(s, 9H, $\left.\mathrm{CH}_{3}\right) ;{ }^{13} \mathrm{C} \mathrm{NMR}\left(100 \mathrm{MHz}, \mathrm{CDCl}_{3}\right) \delta: 189.0$, $171.8,170.8,166.1,165.0,148.5,143.7,141.6,136.7$, $134.8,129.5,128.8,128.4,128.4,128.4,128.3,128.3$, $127.9,127.8,123.6,111.2,101.8,65.3,55.7,51.7,51.3$, $46.3,30.5,21.5$; IR (KBr) v: 2947, 1731, 1664, 1614, $1559,1454,1405,1370,1323,1259,1214,1081,1033$, 916, 822, 743, 697, 593, 548, $\mathrm{cm}^{-1}$; HRMS (ESI) calcd for $\mathrm{C}_{35} \mathrm{H}_{33} \mathrm{ClN}_{2} \mathrm{NaO}_{6}[\mathrm{M}+\mathrm{Na}]^{+}$635.1925, found 635.1923.

Dimethyl 1'-benzyl-2-(tert-butylamino)-5'-fluoro-5-(4methylbenzoyl)-2'-oxospiro[cyclopentane-1,3'-indoline]2,4-diene-3,4-dicarboxylate (1i): Yellow solid, 72\% yield. m.p. 202 $204{ }^{\circ} \mathrm{C} ;{ }^{1} \mathrm{H}$ NMR $\left(400 \mathrm{MHz}, \mathrm{CDCl}_{3}\right) \delta: 10.04$ $(\mathrm{s}, 1 \mathrm{H}, \mathrm{NH}), 7.55(\mathrm{~d}, J=7.6 \mathrm{~Hz}, 2 \mathrm{H}, \mathrm{ArH}), 7.36 \sim 7.34(\mathrm{~m}$, 4H, ArH), 7.30 (d, $J=7.2 \mathrm{~Hz}, 1 \mathrm{H}, \operatorname{ArH}), 7.11$ (d, $J=7.6$ $\mathrm{Hz}, 2 \mathrm{H}, \mathrm{ArH}), 6.93 \sim 6.87(\mathrm{~m}, 2 \mathrm{H}, \mathrm{ArH}), 6.77 \sim 6.74(\mathrm{~m}$, $1 \mathrm{H}, \mathrm{ArH}), 5.02\left(\mathrm{~s}, 2 \mathrm{H}, \mathrm{CH}_{2}\right), 3.72\left(\mathrm{~s}, 3 \mathrm{H}, \mathrm{OCH}_{3}\right), 3.19(\mathrm{~s}$, $\left.3 \mathrm{H}, \mathrm{OCH}_{3}\right), 2.34\left(\mathrm{~s}, 3 \mathrm{H}, \mathrm{CH}_{3}\right), 1.02\left(\mathrm{~s}, 9 \mathrm{H}, \mathrm{CH}_{3}\right) ;{ }^{13} \mathrm{C}$ NMR $\left(100 \mathrm{MHz}, \mathrm{CDCl}_{3}\right) \delta: 189.0,171.9,170.8,166.1$, $165.0,159.1$ (d, $J=248.3 \mathrm{~Hz}), 148.4,141.6,141.2,136.8$, $134.9,128.7,128.5,128.4,128.3,128.1,128.1,127.9$, 127.8, 115.9 (d, $J=23.4 \mathrm{~Hz}), 111.4$ (d, $J=24.9 \mathrm{~Hz}), 110.9$ (d, $J=8.0 \mathrm{~Hz}), 101.8,65.6,55.6,51.6,51.2,46.4,30.5$, 21.5; IR (KBr) v: 2955, 1728, 1656, 1610, 1561, 1485, 1452, 1397, 1324, 1261, 1216, 1033, 919, 819, 693, 637, $597,558 \mathrm{~cm}^{-1}$; HRMS (ESI) calcd for $\mathrm{C}_{35} \mathrm{H}_{33} \mathrm{FN}_{2} \mathrm{NaO}_{6}$ $[\mathrm{M}+\mathrm{Na}]^{+}$619.2220, found 619.2217.

5-Ethyl 3,4-dimethyl 1'-benzyl-2-(tert-butylamino)5'-chloro-2'-oxospiro[cyclopentane-1,3'-indoline]-2,4diene-3,4,5-tricarboxylate (1j): Yellow solid, 75\% yield. m.p. $189 \sim 191{ }^{\circ} \mathrm{C} ;{ }^{1} \mathrm{H}$ NMR (400 MHz, $\left.\mathrm{CDCl}_{3}\right) \delta$ : 9.97 (s, 1H, NH), 7.48 (d, J=7.2 Hz, 2H, ArH), 7.36 7.29 (m, $3 \mathrm{H}, \mathrm{ArH}), 7.24 \sim 7.21(\mathrm{~m}, 1 \mathrm{H}, \mathrm{ArH}), 7.02(\mathrm{~d}, J=1.6 \mathrm{~Hz}$,
1H, ArH), 6.82 (d, $J=8.2 \mathrm{~Hz}, 1 \mathrm{H}, \mathrm{ArH}), 5.02$ (d, $J=15.2$ $\left.\mathrm{Hz}, 1 \mathrm{H}, \mathrm{CH}_{2}\right), 4.83$ (d, J=15.2 Hz, $\left.1 \mathrm{H}, \mathrm{CH}_{2}\right), 4.06 \sim 3.98$ $\left(\mathrm{m}, 1 \mathrm{H}, \mathrm{CH}_{2}\right), 3.89\left(\mathrm{~s}, 3 \mathrm{H}, \mathrm{OCH}_{3}\right), 3.76\left(\mathrm{~s}, 3 \mathrm{H}, \mathrm{OCH}_{3}\right)$, $3.75 \sim 3.71\left(\mathrm{~m}, 1 \mathrm{H}, \mathrm{CH}_{2}\right), 0.98\left(\mathrm{~s}, 9 \mathrm{H}, \mathrm{CH}_{3}\right), 0.95(\mathrm{~d}, J=$ $\left.7.2 \mathrm{~Hz}, 3 \mathrm{H}, \mathrm{CH}_{3}\right) ;{ }^{13} \mathrm{C} \mathrm{NMR}\left(100 \mathrm{MHz}, \mathrm{CDCl}_{3}\right) \delta: 171.4$, $170.6,166.1,165.8,160.7,149.9,143.5,134.7,129.4$, $128.7,128.5,128.4,128.4,128.1,124.1,116.7,110.6$, $100.9,63.9,60.1,55.5,52.3,51.4,45.8,30.5,13.8$; IR (KBr) v: 2976, 1718, 1657, 1572, 1453, 1405, 1365, 1312, 1256, 1215, 1177, 1120, 1024, 968, 806, 746, 694, 604, $546 \mathrm{~cm}^{-1}$; HRMS (ESI) calcd for $\mathrm{C}_{30} \mathrm{H}_{31} \mathrm{ClN}_{2} \mathrm{NaO}_{7}[\mathrm{M}+$ $\mathrm{Na}^{+}$589.1717, found 589.1713.

5-Ethyl 3,4-dimethyl 1'-benzyl-2-(tert-butylamino)-5'methyl-2'-oxospiro[cyclopentane-1,3'-indoline]-2,4-diene3,4,5-tricarboxylate (1k): Yellow solid, 71\% yield. m.p. $144 \sim 146{ }^{\circ} \mathrm{C} ;{ }^{1} \mathrm{H}$ NMR $\left(400 \mathrm{MHz}, \mathrm{CDCl}_{3}\right) \delta: 9.94(\mathrm{~s}, 1 \mathrm{H}$, $\mathrm{NH}), 7.50(\mathrm{~d}, J=7.2 \mathrm{~Hz}, 2 \mathrm{H}, \mathrm{ArH}), 7.35 \sim 7.28(\mathrm{~m}, 3 \mathrm{H}$, ArH), 7.04 (d, J=7.2 Hz, 1H, ArH), 6.84 (s, 1H, ArH), $6.78(\mathrm{~d}, J=8.0 \mathrm{~Hz}, 1 \mathrm{H}, \mathrm{ArH}), 5.02(\mathrm{~d}, J=12.8 \mathrm{~Hz}, 1 \mathrm{H}$, $\left.\mathrm{CH}_{2}\right), 4.79\left(\mathrm{~d}, J=15.2 \mathrm{~Hz}, 1 \mathrm{H}, \mathrm{CH}_{2}\right), 4.03 \sim 3.96(\mathrm{~m}, 1 \mathrm{H}$, $\left.\mathrm{CH}_{2}\right), 3.89\left(\mathrm{~s}, 3 \mathrm{H}, \mathrm{OCH}_{3}\right), 3.76\left(\mathrm{~s}, 3 \mathrm{H}, \mathrm{OCH}_{3}\right), 3.72 \sim 3.68$ $\left(\mathrm{m}, 1 \mathrm{H}, \mathrm{CH}_{2}\right), 2.23\left(\mathrm{~s}, 3 \mathrm{H}, \mathrm{CH}_{3}\right), 0.96\left(\mathrm{~s}, 9 \mathrm{H}, \mathrm{CH}_{3}\right), 0.89$ (t, $\left.J=7.2 \mathrm{~Hz}, 3 \mathrm{H}, \mathrm{CH}_{3}\right) ;{ }^{13} \mathrm{C} \mathrm{NMR}\left(100 \mathrm{MHz}, \mathrm{CDCl}_{3}\right) \delta$ : $171.8,171.5,165.9,160.8,149.5,142.5,135.2,132.8$, 129.7, 128.6, 127.8, 126.5, 124.6, 109.4, 64.3, 59.9, 55.4, 52.2, 51.2, 45.7, 30.5, 20.8, 13.7; IR (KBr) v: 2975, 1748, 1709, 1656, 1573, 1496, 1455 1405, 1365, 1309, 1252, 1214, 1183, 1113, 1024, 969, 802, 696, $611 \mathrm{~cm}^{-1}$; HRMS (ESI) calcd for $\mathrm{C}_{31} \mathrm{H}_{34} \mathrm{~N}_{2} \mathrm{NaO}_{7}[\mathrm{M}+\mathrm{Na}]^{+}$569.2264, found 569.2260 .

Trimethyl 1'-benzyl-2-(tert-butylamino)-2'-oxospiro[cyclopentane-1,3'-indoline]-2,4-diene-3,4,5-tricarboxylate (11): Yellow solid, 69\% yield. m.p. $116 \sim 118{ }^{\circ} \mathrm{C} ;{ }^{1} \mathrm{H}$ NMR $\left(400 \mathrm{MHz}, \mathrm{CDCl}_{3}\right) \delta: 9.97(\mathrm{~s}, 1 \mathrm{H}, \mathrm{NH}), 7.51(\mathrm{~d}, J=7.2 \mathrm{~Hz}$, 2H, ArH), $7.36 \sim 7.29(\mathrm{~m}, 3 \mathrm{H}, \mathrm{ArH}), 7.24 \sim 7.23(\mathrm{~m}, 1 \mathrm{H}$, ArH), $7.02 \sim 6.96(\mathrm{~m}, 2 \mathrm{H}, \mathrm{ArH}), 6.91(\mathrm{~d}, J=8.0 \mathrm{~Hz}, 1 \mathrm{H}$, ArH), $5.11\left(\mathrm{~d}, J=15.2 \mathrm{~Hz}, 1 \mathrm{H}, \mathrm{CH}_{2}\right), 4.76(\mathrm{~d}, J=15.2 \mathrm{~Hz}$, $\left.1 \mathrm{H}, \mathrm{CH}_{2}\right), 3.89\left(\mathrm{~s}, 3 \mathrm{H}, \mathrm{OCH}_{3}\right), 3.76\left(\mathrm{~s}, 3 \mathrm{H}, \mathrm{OCH}_{3}\right), 3.30(\mathrm{~s}$, $\left.3 \mathrm{H}, \mathrm{OCH}_{3}\right), 0.97\left(\mathrm{~s}, 9 \mathrm{H}, \mathrm{CH}_{3}\right) ;{ }^{13} \mathrm{C} \mathrm{NMR}(100 \mathrm{MHz}$, $\left.\mathrm{CDCl}_{3}\right) \delta: 171.8,171.5,165.9,160.8,149.5,142.5,135.2$, $132.8,129.7,128.6,127.8,126.5,124.6,109.4,64.3,59.9$, $55.4,52.2,51.2,45.7,30.5,20.8,13.7$; IR (KBr) v: 2975, $1748,1709,1656,1573,1496,1455$ 1405, 1365, 1309, 1252, 1214, 1183, 1113, 1024, 969, 802, 696, $611 \mathrm{~cm}^{-1}$; HRMS (ESI) calcd for $\mathrm{C}_{29} \mathrm{H}_{30} \mathrm{~N}_{2} \mathrm{NaO}_{7}[\mathrm{M}+\mathrm{Na}]$ 541.1951, found 541.1949.

Trimethyl 1'-benzyl-2-(tert-butylamino)-5'-chloro-2'oxospiro[cyclopentane-1,3'-indoline]-2,4-diene-3,4,5tricarboxylate $(\mathbf{1 m})$ : Yellow solid, 78\% yield. m.p. 220 $222{ }^{\circ} \mathrm{C} ;{ }^{1} \mathrm{H}$ NMR $\left(400 \mathrm{MHz}, \mathrm{CDCl}_{3}\right) \delta: 9.99$ (s, $\left.1 \mathrm{H}, \mathrm{NH}\right)$, 7.48 (d, $J=7.2 \mathrm{~Hz}, 2 \mathrm{H}, \mathrm{ArH}), 7.37 \sim 7.28$ (m, 3H, ArH), $7.23 \sim 7.21(\mathrm{~m}, 1 \mathrm{H}, \mathrm{ArH}), 7.01(\mathrm{~d}, J=2.0 \mathrm{~Hz}, 1 \mathrm{H}, \mathrm{ArH})$, $6.82(\mathrm{~d}, J=8.4 \mathrm{~Hz}, 1 \mathrm{H}, \mathrm{ArH}), 5.08$ (d, $J=15.2 \mathrm{~Hz}, 1 \mathrm{H}$, $\mathrm{CH}_{2}$ ), 4.77 (d, $\left.J=15.2 \mathrm{~Hz}, 1 \mathrm{H}, \mathrm{CH}_{2}\right), 3.90\left(\mathrm{~s}, 3 \mathrm{H}, \mathrm{OCH}_{3}\right)$, $3.76\left(\mathrm{~s}, 3 \mathrm{H}, \mathrm{OCH}_{3}\right), 3.36\left(\mathrm{~s}, 3 \mathrm{H}, \mathrm{OCH}_{3}\right), 1.00\left(\mathrm{~s}, 9 \mathrm{H}, \mathrm{CH}_{3}\right)$; ${ }^{13} \mathrm{C} \mathrm{NMR}\left(100 \mathrm{MHz}, \mathrm{CDCl}_{3}\right) \delta: 171.4,170.6,166.1,165.8$, $161.1,150.2,143.4,134.7,129.5,128.8,128.5,128.5$, 
$128.3,128.1,124.1,116.4,110.6,100.9,63.9,55.6,52.4$, 51.4, 51.3, 45.7, 30.5; IR (KBr) v: 2954, 1754, 1715, 1658, $1578,1441,1370,1321,1215,1118,1036,925,815,744$, $695,602,549 \mathrm{~cm}^{-1}$; HRMS (ESI) calcd for $\mathrm{C}_{29} \mathrm{H}_{29} \mathrm{Cl}-$ $\mathrm{N}_{2} \mathrm{NaO}_{7}[\mathrm{M}+\mathrm{Na}]^{+}$575.1561, found 575.1552.

4.2 General procedure for the synthesis of spiro[cyclopentane-1,3'-indolines] (2a 2e)

A mixture of alkylisocyanide $(0.90 \mathrm{mmol})$, DMAD $(0.90 \mathrm{mmol})$ and 3 -methyleneoxindole $(0.3 \mathrm{mmol})$ in toluene $(10.0 \mathrm{~mL})$ was refluxed at $110{ }^{\circ} \mathrm{C}$ for one hour. Then, the solvent was removed by rotatory evaporation at reduced pressure. The residue was subjected to column chromatography with petroleum ether and ethyl acetate $(V: V=2: 1)$ as eluent to give the pure product.

Dimethyl 2-(cyclohexylamino)-1'-((1Z)-1-(cyclohexylimino)-4-methoxy-2-(methoxycarbonyl)-4-oxobut2-en-1-yl)-5-(4-methoxybenzoyl)-5'-methyl-2'-oxospiro[cyclopentane-1,3'-indoline]-2,4-diene-3,4-dicarboxylate (2a): Yellow solid, 63\% yield. m.p. $220 \sim 222{ }^{\circ} \mathrm{C} ;{ }^{1} \mathrm{H}$ NMR (400 MHz, $\left.\mathrm{CDCl}_{3}\right) \delta: 8.66(\mathrm{~d}, J=9.2 \mathrm{~Hz}, 1 \mathrm{H}, \mathrm{NH})$, 7.98 (d, $J=7.6 \mathrm{~Hz}, 1 \mathrm{H}, \operatorname{ArH}), 7.45$ (d, $J=7.6 \mathrm{~Hz}, 2 \mathrm{H}$, ArH), $7.13 \sim 7.10(\mathrm{~m}, 2 \mathrm{H}, \mathrm{ArH}), 6.90(\mathrm{~s}, 1 \mathrm{H}, \mathrm{CH}), 6.79$ (d, $J=7.2 \mathrm{~Hz}, 2 \mathrm{H}, \mathrm{ArH}), 3.81\left(\mathrm{~s}, 3 \mathrm{H}, \mathrm{OCH}_{3}\right), 3.80(\mathrm{~s}, 3 \mathrm{H}$, $\left.\mathrm{OCH}_{3}\right), 3.68\left(\mathrm{~s}, 3 \mathrm{H}, \mathrm{OCH}_{3}\right), 3.59\left(\mathrm{~s}, 3 \mathrm{H}, \mathrm{OCH}_{3}\right), 3.32(\mathrm{~s}$, $1 \mathrm{H}, \mathrm{CH}), 3.24\left(\mathrm{~s}, 3 \mathrm{H}, \mathrm{OCH}_{3}\right), 2.98(\mathrm{~s}, 1 \mathrm{H}, \mathrm{CH}), 2.27(\mathrm{~s}$, $\left.3 \mathrm{H}, \mathrm{CH}_{3}\right), 1.85 \sim 1.75\left(\mathrm{~m}, 5 \mathrm{H}, \mathrm{CH}_{2}\right), 1.63 \sim 1.59(\mathrm{~m}, 3 \mathrm{H}$, $\left.\mathrm{CH}_{2}\right), 1.52 \sim 1.46\left(\mathrm{~m}, 3 \mathrm{H}, \mathrm{CH}_{2}\right), 1.34 \sim 1.28\left(\mathrm{~m}, 4 \mathrm{H}, \mathrm{CH}_{2}\right)$, $1.15 \sim 1.09\left(\mathrm{~m}, 1 \mathrm{H}, \mathrm{CH}_{2}\right), 0.96 \sim 0.90\left(\mathrm{~m}, 4 \mathrm{H}, \mathrm{CH}_{2}\right) ;{ }^{13} \mathrm{C}$ NMR $\left(100 \mathrm{MHz}, \mathrm{CDCl}_{3}\right) \delta: 186.7,171.3,170.6,165.6$, $165.2,164.1,163.6,162.1,147.6,144.1,140.7,137.9$, $133.5,131.8,130.5,129.9,129.7,127.8,125.7,122.7$, $115.5,112.8,98.5,64.9,59.9,55.3,54.6,52.6,52.5,51.7$, $50.9,34.2,33.9,33.5,33.2,25.8,24.9,24.8,24.2,23.9$, 21.1; IR (KBr) v: 3442, 3246, 2937, 2855, 1735, 1667, $1598,1553,1489,1460,1332,1249,1170,1093,1042$, 925, 821, 792, 700, 602, $546 \mathrm{~cm}^{-1}$; HRMS (ESI) calcd for $\mathrm{C}_{44} \mathrm{H}_{49} \mathrm{~N}_{3} \mathrm{NaO}_{11}[\mathrm{M}+\mathrm{Na}]^{+}$818.3265, found 818.3254.

Dimethyl 2-(cyclohexylamino)-1'-((1Z)-1-(cyclohexylimino)-4-methoxy-2-(methoxycarbonyl)-4-oxobut-2en-1-yl)-5-(4-methylbenzoyl)-2'-oxospiro[cyclopentane1,3'-indoline]-2,4-diene-3,4-dicarboxylate (2b): Yellow solid, 59\% yield. m.p. 209 211 ${ }^{\circ} \mathrm{C} ;{ }^{1} \mathrm{H}$ NMR $(400 \mathrm{MHz}$, $\left.\mathrm{CDCl}_{3}\right) \delta: 8.69(\mathrm{~d}, J=10.4 \mathrm{~Hz}, 1 \mathrm{H}, \mathrm{NH}), 8.10(\mathrm{~d}, J=8.0$ $\mathrm{Hz}, 1 \mathrm{H}, \mathrm{ArH}), 7.35 \sim 7.32(\mathrm{~m}, 3 \mathrm{H}, \mathrm{ArH}, \mathrm{CH}), 7.11 \sim 7.03$ $(\mathrm{m}, 5 \mathrm{H}, \mathrm{ArH}), 3.82\left(\mathrm{~s}, 3 \mathrm{H}, \mathrm{OCH}_{3}\right), 3.67\left(\mathrm{~s}, 3 \mathrm{H}, \mathrm{OCH}_{3}\right)$, $3.59\left(\mathrm{~s}, 3 \mathrm{H}, \mathrm{OCH}_{3}\right), 3.35 \sim 3.31(\mathrm{~m}, 1 \mathrm{H}, \mathrm{CH}), 3.17(\mathrm{~s}, 3 \mathrm{H}$, $\left.\mathrm{OCH}_{3}\right), 2.96 \sim 2.98(\mathrm{~m}, 1 \mathrm{H}, \mathrm{CH}), 2.32\left(\mathrm{~s}, 3 \mathrm{H}, \mathrm{CH}_{3}\right)$, $1.85 \sim 1.76\left(\mathrm{~m}, 5 \mathrm{H}, \mathrm{CH}_{2}\right), 1.64 \sim 1.58\left(\mathrm{~m}, 4 \mathrm{H}, \mathrm{CH}_{2}\right)$, $1.53 \sim 1.45\left(\mathrm{~m}, 3 \mathrm{H}, \mathrm{CH}_{2}\right), 1.35 \sim 1.26\left(\mathrm{~m}, 4 \mathrm{H}, \mathrm{CH}_{2}\right)$, $1.15 \sim 1.07\left(\mathrm{~m}, 1 \mathrm{H}, \mathrm{CH}_{2}\right), 0.95 \sim 0.85\left(\mathrm{~m}, 3 \mathrm{H}, \mathrm{CH}_{2}\right) ;{ }^{13} \mathrm{C}$ NMR $\left(100 \mathrm{MHz}, \mathrm{CDCl}_{3}\right) \delta: 187.5,171.3,170.5,165.6$, $164.9,164.1,163.5,148.2,144.1,143.1,141.5,137.8$, $136.3,130.0,129.2,128.4,128.2,127.7,125.8,123.9$, $122.2,115.8,98.7,64.7,59.9,54.8,52.6,52.6,51.6,51.0$, $34.2,33.9,33.5,33.1,25.8,24.9,24.8,24.2,23.9,21.5$; IR (KBr) $v: 3731,3441,2933,2856,1734,1657,1600,1554$,
1463, 1357, 1308, 1255, 1216, 1156, 1087, 1049, 894, 792, $750,707,59,512 \mathrm{~cm}^{-1}$; HRMS (ESI) calcd for $\mathrm{C}_{43} \mathrm{H}_{47} \mathrm{~N}_{3}$ $\mathrm{NaO}_{10}[\mathrm{M}+\mathrm{Na}]^{+} 788.3159$, found 788.3161 .

Dimethyl 2-(tert-butylamino)-1'-((1Z)-1-(tert-butylimino)-4-methoxy-2-(methoxycarbonyl)-4-oxobut-2-en-1yl)-5'-chloro-5-(4-methylbenzoyl)-2'-oxospiro[cyclopentane-1,3'-indoline]-2,4-diene-3,4-dicarboxylate (2c): Yellow solid, $70 \%$ yield. m.p. 212 $214{ }^{\circ} \mathrm{C} ;{ }^{1} \mathrm{H}$ NMR (400 $\left.\mathrm{MHz}, \mathrm{CDCl}_{3}\right) \delta: 9.99(\mathrm{~s}, 1 \mathrm{H}, \mathrm{NH}), 7.99(\mathrm{~d}, J=8.4 \mathrm{~Hz}, 1 \mathrm{H}$, $\mathrm{ArH}), 7.30 \sim 7.28(\mathrm{~m}, 3 \mathrm{H}, \mathrm{ArH}, \mathrm{CH}), 7.10 \sim 7.05(\mathrm{~m}, 4 \mathrm{H}$, ArH), $3.83\left(\mathrm{~s}, 3 \mathrm{H}, \mathrm{OCH}_{3}\right), 3.67\left(\mathrm{~s}, 3 \mathrm{H}, \mathrm{OCH}_{3}\right), 3.60(\mathrm{~s}, 3 \mathrm{H}$, $\left.\mathrm{OCH}_{3}\right), 3.15\left(\mathrm{~s}, 3 \mathrm{H}, \mathrm{OCH}_{3}\right), 2.33\left(\mathrm{~s}, 3 \mathrm{H}, \mathrm{CH}_{3}\right), 1.33(\mathrm{~s}, 9 \mathrm{H}$, $\left.\mathrm{CH}_{3}\right), 1.11\left(\mathrm{~s}, 9 \mathrm{H}, \mathrm{CH}_{3}\right) ;{ }^{13} \mathrm{C} \mathrm{NMR}\left(100 \mathrm{MHz}, \mathrm{CDCl}_{3}\right) \delta$ : $187.6,171.1,170.5,166.1,164.9,164.4,163.9,147.1$, $142.3,141.7,139.9,139.4,136.3,129.7,129.3,128.8$, $128.5,128.2,127.7,122.8,117.3,100.9,64.8,57.0,55.9$, $52.5,52.4,51.6,51.2,30.6,30.5,30.5,21.5$; IR (KBr) $v$ : 3681, 3091, 2964, 1740, 1668, 1614, 1562, 1466, 1399, $1359,1331,1255,1212,1084,1036,909,821,770,687$, 594, $540 \mathrm{~cm}^{-1}$; HRMS (ESI) calcd for $\mathrm{C}_{39} \mathrm{H}_{42} \mathrm{ClN}_{3} \mathrm{NaO}_{10}$ $[\mathrm{M}+\mathrm{Na}]^{+}$770.2456, found 770.2445 .

Dimethyl 2-(tert-butylamino)-1'-((1Z)-1-(tert-butylimino)-4-methoxy-2-(methoxycarbonyl)-4-oxobut-2-en-1yl)-5-(4-chlorobenzoyl)-5'-methyl-2'-oxospiro[cyclopentane-1,3'-indoline]-2,4-diene-3,4-dicarboxylate (2d): Yellow solid, 64\% yield. m.p. 197 $199{ }^{\circ} \mathrm{C} ;{ }^{1} \mathrm{H}$ NMR $(400$ $\left.\mathrm{MHz}, \mathrm{CDCl}_{3}\right) \delta: 10.03(\mathrm{~s}, 1 \mathrm{H}, \mathrm{NH}), 7.93$ (d, $J=8.4 \mathrm{~Hz}$, $1 \mathrm{H}, \mathrm{ArH}), 7.33 \sim 7.29(\mathrm{~m}, 3 \mathrm{H}, \mathrm{ArH}, \mathrm{CH}), 7.24(\mathrm{~s}, 1 \mathrm{H}$, ArH), 7.12 (d, J=8.0 Hz, 1H, ArH), 7.02(s, 1H, ArH), $6.87(\mathrm{~s}, 1 \mathrm{H}, \mathrm{ArH}), 3.83\left(\mathrm{~s}, 3 \mathrm{H}, \mathrm{OCH}_{3}\right), 3.68\left(\mathrm{~s}, 3 \mathrm{H}, \mathrm{OCH}_{3}\right)$, $3.58\left(\mathrm{~s}, 3 \mathrm{H}, \mathrm{OCH}_{3}\right), 3.20\left(\mathrm{~s}, 3 \mathrm{H}, \mathrm{OCH}_{3}\right), 2.27\left(\mathrm{~s}, 3 \mathrm{H}, \mathrm{CH}_{3}\right)$, $1.34\left(\mathrm{~s}, 9 \mathrm{H}, \mathrm{CH}_{3}\right), 1.09$ (s, 9H, $\left.\mathrm{CH}_{3}\right) ;{ }^{13} \mathrm{C} \mathrm{NMR} \mathrm{(100} \mathrm{MHz,}$ $\left.\mathrm{CDCl}_{3}\right) \delta: 186.4,171.7,171.6,166.0,165.2,164.3,164.1$, $147.7,141.4,140.1,139.4,137.6,137.1,133.4,129.9$, $129.8,129.4,128.1,127.7,125.7,123.2,115.9,100.7$, 65.2, 56.9, 55.9, 52.5, 52.4, 51.7, 51.2, 30.5, 30.4, 20.9; IR (KBr) v: 3685, 3087, 2957, 1737, 1673, 1559, 1460, 1398, 1359, 1320, 1255, 1220, 1186, 1083, 1024, 912, 813, 677, $536 \mathrm{~cm}^{-1}$; HRMS (ESI) calcd for $\mathrm{C}_{39} \mathrm{H}_{42} \mathrm{ClN}_{3} \mathrm{NaO}_{10}[\mathrm{M}+$ $\mathrm{Na}]^{+}$770.2456, found 770.2449.

Dimethyl 2-(tert-butylamino)-1'-((1Z)-1-(tert-butylimino)-4-methoxy-2-(methoxycarbonyl)-4-oxobut-2-en-1yl)-5'-fluoro-5-(4-methoxybenzoyl)-2'-oxospiro[cyclopentane-1,3'-indoline]-2,4-diene-3,4-dicarboxylate (2e): Yellow solid, $67 \%$ yield. m.p. $218 \sim 220{ }^{\circ} \mathrm{C} ;{ }^{1} \mathrm{H}$ NMR (400 $\left.\mathrm{MHz}, \mathrm{CDCl}_{3}\right) \delta: 9.95(\mathrm{~s}, 1 \mathrm{H}, \mathrm{NH}), 8.04 \sim 8.01(\mathrm{~m}, 1 \mathrm{H}$, ArH), 7.39 (d, J=8.8 Hz, 2H, ArH, CH), $7.05 \sim 7.04(\mathrm{~m}$, $1 \mathrm{H}, \mathrm{ArH}), 7.01(\mathrm{~d}, J=2.8 \mathrm{~Hz}, 1 \mathrm{H}, \mathrm{ArH}), 6.80 \sim 6.77(\mathrm{~m}$, $3 \mathrm{H}, \mathrm{ArH}), 3.83\left(\mathrm{~s}, 3 \mathrm{H}, \mathrm{OCH}_{3}\right), 3.80\left(\mathrm{~s}, 3 \mathrm{H}, \mathrm{OCH}_{3}\right), 3.68(\mathrm{~s}$, $\left.3 \mathrm{H}, \mathrm{OCH}_{3}\right), 3.61\left(\mathrm{~s}, 3 \mathrm{H}, \mathrm{OCH}_{3}\right), 3.21\left(\mathrm{~s}, 3 \mathrm{H}, \mathrm{OCH}_{3}\right), 1.33$ $\left(\mathrm{s}, 9 \mathrm{H}, \mathrm{CH}_{3}\right), 1.11\left(\mathrm{~s}, 9 \mathrm{H}, \mathrm{CH}_{3}\right) ;{ }^{13} \mathrm{C} \mathrm{NMR}(100 \mathrm{MHz}$, $\left.\mathrm{CDCl}_{3}\right) \delta: 186.8,171.2,170.3,166.1,165.1,164.4,163.9$, $162.2,159.2$ (d, $J=242.1 \mathrm{~Hz}), 146.3,139.9,139.8,139.6$, $131.7,130.6,129.5,128.6,127.3,127.3,117.3$ (d, $J=7.6$ $\mathrm{Hz}), 115.9$ (d, $J=22.7 \mathrm{~Hz}), 112.8,110.2$ (d, $J=24.7 \mathrm{~Hz})$, $100.9,65.1,56.9,55.8,55.3,52.5,52.4,51.7,51.1,30.5$, 30.5; IR (KBr) v: 3684, 3095, 2965, 1739, 1670, 1601, 
$1565,1472,1451,1402,1361,1312,1255,1214,1176$, 1078, 1033, 902, 829, 687, 598, $526 \mathrm{~cm}^{-1}$; HRMS (ESI) calcd for $\mathrm{C}_{39} \mathrm{H}_{42} \mathrm{FN}_{3} \mathrm{NaO}_{11}[\mathrm{M}+\mathrm{Na}]^{+} 770.2701$, found 770.2689 .

\subsection{General procedure for the synthesis of spi-} ro[cyclopentane-1,3'-indolines] (3a $\mathbf{3 h}$ )

A mixture of alkylisocyanide $(0.45 \mathrm{mmol})$, alkyl propiolate $(0.45 \mathrm{mmol})$ and 3 -methyleneoxindole $(0.3 \mathrm{mmol})$ in toluene $(10.0 \mathrm{~mL})$ was refluxed at $110{ }^{\circ} \mathrm{C}$ for $3 \mathrm{~h}$. Then, the solvent was removed by rotatory evaporation at reduced pressure. The residue was subjected to column chromatography with petroleum ether and ethyl acetate $(V: V=2: 1)$ as eluent to give the pure product.

Methyl 1'-benzyl-5-(tert-butylamino)-5'-fluoro-2-(4methoxybenzoyl)-2'-oxospiro[cyclopentane-1,3'-indoline]2,4-diene-3-carboxylate (3a): Orange solid, 88\% yield. m.p. $192 \sim 194{ }^{\circ} \mathrm{C} ;{ }^{1} \mathrm{H}$ NMR $\left(400 \mathrm{MHz}, \mathrm{CDCl}_{3}\right) \delta$ : 7.59 (d, $J=8.8 \mathrm{~Hz}, 2 \mathrm{H}, \mathrm{ArH}), 7.44$ (d, $J=7.6 \mathrm{~Hz}, 2 \mathrm{H}, \mathrm{ArH}), 7.35$ $(\mathrm{t}, J=7.2 \mathrm{~Hz}, 2 \mathrm{H}, \mathrm{ArH}), 7.30 \sim 7.29(\mathrm{~m}, 1 \mathrm{H}, \mathrm{ArH}), 6.86$ $(\mathrm{d}, J=8.4 \mathrm{~Hz}, 3 \mathrm{H}, \mathrm{ArH}), 6.77 \sim 6.75(\mathrm{~m}, 1 \mathrm{H}, \mathrm{ArH}), 6.72 \sim$ $6.69(\mathrm{~m}, 1 \mathrm{H}, \mathrm{ArH}), 5.50(\mathrm{~s}, 1 \mathrm{H}, \mathrm{CH}), 5.26(\mathrm{~d}, J=15.6 \mathrm{~Hz}$, $\left.1 \mathrm{H}, \mathrm{CH}_{2}\right), 4.77\left(\mathrm{~d}, J=16.0 \mathrm{~Hz}, 1 \mathrm{H}, \mathrm{CH}_{2}\right), 3.82(\mathrm{~s}, 3 \mathrm{H}$, $\left.\mathrm{OCH}_{3}\right), 3.67(\mathrm{~s}, 1 \mathrm{H}, \mathrm{NH}), 3.22\left(\mathrm{~s}, 3 \mathrm{H}, \mathrm{OCH}_{3}\right), 1.24(\mathrm{~s}, 9 \mathrm{H}$, $\left.\mathrm{CH}_{3}\right) ;{ }^{13} \mathrm{C} \mathrm{NMR}\left(100 \mathrm{MHz}, \mathrm{CDCl}_{3}\right) \delta: 187.1,172.1,165.4$, $162.2,160.4,159.1(\mathrm{~d}, J=228.6 \mathrm{~Hz}), 158.77,151.9,140.1$, $135.9,133.3,130.9,130.0,128.9,128.3,128.2,127.8$, $127.5,115.8(\mathrm{~d}, J=23.4 \mathrm{~Hz}), 113.4,111.2(\mathrm{~d}, J=24.8 \mathrm{~Hz})$, 110.5 (d, $J=8.2 \mathrm{~Hz}), 99.7,68.7,55.4,52.5,51.6,44.6$, 28.5; IR (KBr) v: 3398, 3065, 2981, 2943, 2842, 1722, $1601,1529,1489,1451,1360,1256,1142,1073,1022$, 936, 901, 877, 849, 823, 794, 762, 742, 698, 619, 559, 523 $\mathrm{cm}^{-1}$; HRMS (ESI) calcd for $\mathrm{C}_{33} \mathrm{H}_{32} \mathrm{FN}_{2} \mathrm{O}_{5}[\mathrm{M}+\mathrm{H}]^{+}$ 555.2295, found 555.2276.

Methyl 1'-benzyl-5-(tert-butylamino)-5'-fluoro-2-(4methylbenzoyl)-2'-oxospiro[cyclopentane-1,3'-indoline]2,4-diene-3-carboxylate (3b): Orange solid, 83\% yield. m.p. 201 203 ${ }^{\circ} \mathrm{C} ;{ }^{1} \mathrm{H}$ NMR (400 MHz, $\mathrm{CDCl}_{3}$ ) $\delta: 7.49$ (d, $J=7.6 \mathrm{~Hz}, 2 \mathrm{H}, \mathrm{ArH}), 7.45$ (d, $J=7.2 \mathrm{~Hz}, 2 \mathrm{H}, \mathrm{ArH})$, 7.35 (t, $J=7.6 \mathrm{~Hz}, 2 \mathrm{H}, \mathrm{ArH}), 7.31 \sim 7.29$ (m, 1H, ArH), $7.17(\mathrm{~d}, J=8.0 \mathrm{~Hz}, 2 \mathrm{H}, \mathrm{ArH}), 6.91 \sim 6.86(\mathrm{~m}, 1 \mathrm{H}, \mathrm{ArH})$, $6.77 \sim 6.75(\mathrm{~m}, 1 \mathrm{H}, \mathrm{ArH}), 6.73 \sim 6.69(\mathrm{~m}, 1 \mathrm{H}, \mathrm{ArH}), 5.49$ $(\mathrm{s}, 1 \mathrm{H}, \mathrm{CH}), 5.26\left(\mathrm{~d}, J=15.6 \mathrm{~Hz}, 1 \mathrm{H}, \mathrm{CH}_{2}\right), 4.78(\mathrm{~d}, J=$ $\left.15.6 \mathrm{~Hz}, 1 \mathrm{H}, \mathrm{CH}_{2}\right), 3.71(\mathrm{~s}, 1 \mathrm{H}, \mathrm{NH}), 3.14$ (s, $\left.3 \mathrm{H}, \mathrm{OCH}_{3}\right)$, $2.35\left(\mathrm{~s}, 3 \mathrm{H}, \mathrm{CH}_{3}\right), 1.24\left(\mathrm{~s}, 9 \mathrm{H}, \mathrm{CH}_{3}\right) ;{ }^{13} \mathrm{C} \mathrm{NMR}(100 \mathrm{MHz}$, $\left.\mathrm{CDCl}_{3}\right) \delta: 187.9,172.1,165.4,159.2,159.2(\mathrm{~d}, J=240.3$ $\mathrm{Hz}), 152.7,141.7,140.1,140.1,137.9,135.9,130.6$, 128.9, $128.8,128.5,127.9,127.8,127.5,115.8(\mathrm{~d}, J=23.4 \mathrm{~Hz})$, $111.1(\mathrm{~d}, J=24.8 \mathrm{~Hz}), 110.5(\mathrm{~d}, J=8.5 \mathrm{~Hz}), 99.7,68.6$, 52.5, 51.5, 44.6, 28.5, 21.5; IR (KBr) v: 3397, 3063, 2976, $1725,1607,1525,1491,1451,1362,1327,1210,1142$, 1076, 1033, 903, 876, 820, 794, 737, 699, 613, 560, 527 $\mathrm{cm}^{-1}$; HRMS (ESI) calcd for $\mathrm{C}_{33} \mathrm{H}_{32} \mathrm{FN}_{2} \mathrm{O}_{4}[\mathrm{M}+\mathrm{H}]^{+}$ 539.2346, found 539.2344.

Methyl 1'-benzyl-5-(tert-butylamino)-2-(4-methoxybenzoyl)-2'-oxospiro[cyclopentane-1,3'-indoline]-2,4-diene-3carboxylate (3c): Orange solid, 76\% yield. m.p. 178
$180{ }^{\circ} \mathrm{C} ;{ }^{1} \mathrm{H}$ NMR (400 MHz, $\left.\mathrm{CDCl}_{3}\right) \delta: 7.60(\mathrm{~d}, J=8.0$ $\mathrm{Hz}, 2 \mathrm{H}, \mathrm{ArH}), 7.46$ (d, $J=6.8 \mathrm{~Hz}, 2 \mathrm{H}, \mathrm{ArH}), 7.36 \sim 7.29$ $(\mathrm{m}, 3 \mathrm{H}, \mathrm{ArH}), 7.19 \sim 7.15(\mathrm{~m}, 1 \mathrm{H}, \mathrm{ArH}), 7.00 \sim 6.98(\mathrm{~m}$, $1 \mathrm{H}, \mathrm{ArH}), 6.95 \sim 6.94(\mathrm{~m}, 1 \mathrm{H}, \mathrm{ArH}), 6.85(\mathrm{~d}, J=7.8 \mathrm{~Hz}$, 2H, ArH), 6.79 (d, J=7.2 Hz, 1H, ArH), 5.50 (s, 1H, CH), $5.28\left(\mathrm{~d}, J=15.6 \mathrm{~Hz}, 1 \mathrm{H}, \mathrm{CH}_{2}\right), 4.78(\mathrm{~d}, J=15.6 \mathrm{~Hz}, 1 \mathrm{H}$, $\left.\mathrm{CH}_{2}\right), 3.82\left(\mathrm{~s}, 3 \mathrm{H}, \mathrm{OCH}_{3}\right), 3.66(\mathrm{~s}, 1 \mathrm{H}, \mathrm{NH}), 3.21(\mathrm{~s}, 3 \mathrm{H}$, $\left.\mathrm{OCH}_{3}\right), 1.22\left(\mathrm{~s}, 9 \mathrm{H}, \mathrm{CH}_{3}\right) ;{ }^{13} \mathrm{C} \mathrm{NMR}\left(100 \mathrm{MHz}, \mathrm{CDCl}_{3}\right) \delta$ : $187.3,172.3,165.6,162.2,159.2,151.6,144.1,136.3$, $133.4,131.4,130.0,129.4,128.8,127.7,127.5,126.6$, $123.2,123.1,113.3,109.9,99.4,68.7,55.4,52.3,51.6$, 44.5, 28.5; IR (KBr) v: 3359, 2974, 2894, 1924, 1709, $1604,1482,1446,1405,1379,1313,1265,1088,1049$, $881,875,806,745,721,671,618,572,512 \mathrm{~cm}^{-1}$; HRMS (ESI) calcd for $\mathrm{C}_{33} \mathrm{H}_{33} \mathrm{~N}_{2} \mathrm{O}_{5}[\mathrm{M}+\mathrm{H}]^{+}$537.2389, found 537.2384 .

Methyl 1'-benzyl-5-(tert-butylamino)-5'-chloro-2-(4chlorobenzoyl)-2'-oxospiro[cyclopentane-1,3'-indoline]2,4-diene-3-carboxylate (3d): Orange solid, 72\% yield. m.p. $183 \sim 185{ }^{\circ} \mathrm{C} ;{ }^{1} \mathrm{H}$ NMR $\left(400 \mathrm{MHz}, \mathrm{CDCl}_{3}\right) \delta: 7.51(\mathrm{~s}$, 1H, ArH), $7.49 \sim 7.47$ (m, 2H, ArH), 7.46 (s, 1H, ArH), $7.34(\mathrm{t}, J=7.2 \mathrm{~Hz}, 2 \mathrm{H}, \mathrm{ArH}), 7.30 \sim 7.28(\mathrm{~m}, 1 \mathrm{H}, \mathrm{ArH})$, $7.16 \sim 7.15(\mathrm{~m}, 3 \mathrm{H}, \mathrm{ArH}), 7.01 \sim 6.99(\mathrm{~m}, 1 \mathrm{H}, \mathrm{ArH})$, $6.96 \sim 6.92(\mathrm{~m}, 1 \mathrm{H}, \mathrm{ArH}), 6.79(\mathrm{~d}, J=7.6 \mathrm{~Hz}, 1 \mathrm{H}, \mathrm{ArH})$, $5.50(\mathrm{~s}, 1 \mathrm{H}, \mathrm{CH}), 5.28\left(\mathrm{~d}, J=15.6 \mathrm{~Hz}, 1 \mathrm{H}, \mathrm{CH}_{2}\right), 4.79$ (d, $\left.J=15.2 \mathrm{~Hz}, 1 \mathrm{H}, \mathrm{CH}_{2}\right), 3.70(\mathrm{~s}, 1 \mathrm{H}, \mathrm{NH}), 3.13(\mathrm{~s}, 3 \mathrm{H}$, $\left.\mathrm{OCH}_{3}\right), 2.34\left(\mathrm{~s}, 3 \mathrm{H}, \mathrm{CH}_{3}\right), 1.22\left(\mathrm{~s}, 9 \mathrm{H}, \mathrm{CH}_{3}\right) ;{ }^{13} \mathrm{C} \mathrm{NMR}$ $\left(100 \mathrm{MHz}, \mathrm{CDCl}_{3}\right) \delta: 188.0,172.3,165.6,159.6,152.2$, $144.2,141.5,138.0,136.3,131.2,129.3,128.8,128.7$, $127.9,127.7,127.5,126.7,123.2,123.1,109.9,99.5,68.6$, 52.4, 51.4, 44.5, 28.5, 21.5; IR (KBr) v: 3397, 3060, 2975, 2944, 1722, 1607, 1522, 1461, 1438, 1367, 1333, 1254, 1210, 1178, 1145, 1079, 1036, 970, 906, 852, 756, 701, $669,626,600,556 \mathrm{~cm}^{-1}$; HRMS (ESI) calcd for $\mathrm{C}_{33} \mathrm{H}_{33}-$ $\mathrm{N}_{2} \mathrm{O}_{4}\left([\mathrm{M}+\mathrm{H}]^{+}\right): 521.2440$, found 521.2437.

Methyl 1'-benzyl-5-(tert-butylamino)-5'-chloro-2-(4chlorobenzoyl)-2'-oxospiro[cyclopentane-1,3'-indoline]2,4-diene-3-carboxylate (3e): Orange solid, $82 \%$ yield. m.p. $226 \sim 228{ }^{\circ} \mathrm{C} ;{ }^{1} \mathrm{H}$ NMR (400 MHz, $\left.\mathrm{CDCl}_{3}\right) \delta: 7.52(\mathrm{~d}$, $J=8.4 \mathrm{~Hz}, 2 \mathrm{H}, \mathrm{ArH}), 7.44(\mathrm{~d}, J=7.2 \mathrm{~Hz}, 2 \mathrm{H}, \mathrm{ArH})$, $7.36 \sim 7.34(\mathrm{~m}, 4 \mathrm{H}, \mathrm{ArH}), 7.31 \sim 7.29(\mathrm{~m}, 1 \mathrm{H}, \mathrm{ArH}), 7.17$ (d, $J=8.0 \mathrm{~Hz}, 2 \mathrm{H}, \mathrm{ArH}), 6.98(\mathrm{~s}, 1 \mathrm{H}, \mathrm{ArH}), 6.73$ (d, $J=$ $8.4 \mathrm{~Hz}, 1 \mathrm{H}, \mathrm{ArH}), 5.50(\mathrm{~s}, 1 \mathrm{H}, \mathrm{CH}), 5.23(\mathrm{~d}, J=15.6 \mathrm{~Hz}$, $\left.1 \mathrm{H}, \mathrm{CH}_{2}\right), 4.79\left(\mathrm{~d}, J=15.6 \mathrm{~Hz}, 1 \mathrm{H}, \mathrm{CH}_{2}\right), 3.80(\mathrm{~s}, 1 \mathrm{H}$, $\mathrm{NH}), 3.22\left(\mathrm{~s}, 3 \mathrm{H}, \mathrm{OCH}_{3}\right), 1.24\left(\mathrm{~s}, 9 \mathrm{H}, \mathrm{CH}_{3}\right) ;{ }^{13} \mathrm{C} \mathrm{NMR}$ $\left(100 \mathrm{MHz}, \mathrm{CDCl}_{3}\right) \delta: 186.5,171.8,165.1,160.1,153.8$, $142.7,138.9,137.3,135.7,129.6,129.5,129.2,128.9$, $128.5,128.5,128.4,127.9,127.5,123.4,110.9,100.1$, 68.1, 52.8, 51.7, 44.6, 28.4; IR (KBr) v: 3400, 3060, 2973, $1728,1611,1521,1486,1457,1429,1398,1365,1331$, 1253, 1208, 1178, 1142, 1089, 1014, 902, 850, 817, 741, $699,552,521 \mathrm{~cm}^{-1}$; HRMS (ESI) calcd for $\mathrm{C}_{32} \mathrm{H}_{29} \mathrm{Cl}_{2} \mathrm{~N}_{2} \mathrm{O}_{4}$ $[\mathrm{M}+\mathrm{H}]^{+}$575.1504, found 575.1503.

Methyl 2-benzoyl-1'-butyl-5-(tert-butylamino)-5'-fluoro2'-oxospiro[cyclopentane-1,3'-indoline]-2,4-diene-3-carboxylate (3f): Orange solid, $65 \%$ yield. m.p. $176 \sim 178{ }^{\circ} \mathrm{C}$; ${ }^{1} \mathrm{H}$ NMR (400 MHz, $\left.\mathrm{CDCl}_{3}\right) \delta: 7.55 \sim 7.52(\mathrm{~m}, 2 \mathrm{H}, \mathrm{ArH})$, 
$7.43 \sim 7.39(\mathrm{~m}, 1 \mathrm{H}, \mathrm{ArH}), 7.37 \sim 7.33(\mathrm{~m}, 2 \mathrm{H}, \mathrm{ArH})$, $7.03 \sim 6.98(\mathrm{~m}, 1 \mathrm{H}, \mathrm{ArH}), 6.88 \sim 6.85(\mathrm{~m}, 1 \mathrm{H}, \mathrm{ArH})$, $6.79 \sim 6.76(\mathrm{~m}, 1 \mathrm{H}, \mathrm{ArH}), 5.48(\mathrm{~s}, 1 \mathrm{H}, \mathrm{CH}), 3.83 \sim 3.79$ $\left(\mathrm{m}, 3 \mathrm{H}, \mathrm{NH}, \mathrm{CH}_{2}\right), 3.09\left(\mathrm{~s}, 3 \mathrm{H}, \mathrm{OCH}_{3}\right), 1.82 \sim 1.75(\mathrm{~m}$, $\left.2 \mathrm{H}, \mathrm{CH}_{2}\right), 1.49 \sim 1.43\left(\mathrm{~m}, 2 \mathrm{H}, \mathrm{CH}_{2}\right), 1.26\left(\mathrm{~s}, 9 \mathrm{H}, \mathrm{CH}_{3}\right)$, $0.99\left(\mathrm{t}, J=7.6 \mathrm{~Hz}, 3 \mathrm{H}, \mathrm{CH}_{3}\right) ;{ }^{13} \mathrm{C} \mathrm{NMR}\left(100 \mathrm{MHz}, \mathrm{CDCl}_{3}\right)$ $\delta: 188.0,171.8,165.4,159.5,159.0(\mathrm{~d}, J=240.2 \mathrm{~Hz})$, $153.1,140.7,140.6,131.0,130.4,128.1,127.7,115.7$ (d, $J=23.3 \mathrm{~Hz}), 111.1(\mathrm{~d}, J=24.7 \mathrm{~Hz}), 109.7(\mathrm{~d}, J=8.0 \mathrm{~Hz})$, 99.9, 68.5, 52.5, 51.4, 40.7, 29.0, 28.5, 20.2, 13.7; IR (KBr) v: 3397, 3120, 2965, 1866, 1789, 1484, 1406, 1297, 1276, 1205, 1179, 1162, 1078, 1021, 956, 902, 841, 652, $585,513 \mathrm{~cm}^{-1}$; HRMS (ESI) calcd for $\mathrm{C}_{29} \mathrm{H}_{31} \mathrm{FN}_{2} \mathrm{NaO}_{4}$ $[\mathrm{M}+\mathrm{Na}]^{+}$513.2166, found 513.2159.

Ethyl 1'-benzyl-5-(tert-butylamino)-5'-chloro-2-(4methoxybenzoyl)-2'-oxospiro[cyclopentane-1,3'-indoline]2,4-diene-3-carboxylate (3g): Yellow solid, 72\% yield. m.p. $202 \sim 204{ }^{\circ} \mathrm{C} ;{ }^{1} \mathrm{H}$ NMR $\left(400 \mathrm{MHz}, \mathrm{CDCl}_{3}\right) \delta: 7.56(\mathrm{~d}$, $J=8.8 \mathrm{~Hz}, 2 \mathrm{H}, \mathrm{ArH}), 7.42(\mathrm{~d}, J=2.0 \mathrm{~Hz}, 1 \mathrm{H}, \mathrm{ArH})$, $7.32 \sim 7.30(\mathrm{~m}, 2 \mathrm{H}, \mathrm{ArH}), 7.27(\mathrm{~s}, 1 \mathrm{H}, \mathrm{ArH}), 7.25(\mathrm{~s}, 1 \mathrm{H}$, ArH), $6.86 \sim 6.84(\mathrm{~m}, 2 \mathrm{H}, \mathrm{ArH}), 6.70(\mathrm{~d}, J=8.8 \mathrm{~Hz}, 2 \mathrm{H}$, $\operatorname{ArH}), 6.21(\mathrm{~d}, J=8.8 \mathrm{~Hz}, 1 \mathrm{H}, \operatorname{ArH}), 5.37$ (d, $J=2.0 \mathrm{~Hz}$, $1 \mathrm{H}, \mathrm{CH}), 5.30\left(\mathrm{~d}, J=16.0 \mathrm{~Hz}, 1 \mathrm{H}, \mathrm{CH}_{2}\right), 4.42(\mathrm{~d}, J=16.0$ $\left.\mathrm{Hz}, 1 \mathrm{H}, \mathrm{CH}_{2}\right), 4.33 \sim 4.28\left(\mathrm{~m}, 2 \mathrm{H}, \mathrm{CH}_{2}\right), 3.79(\mathrm{~s}, 3 \mathrm{H}$, $\left.\mathrm{OCH}_{3}\right), 1.29\left(\mathrm{t}, J=7.2 \mathrm{~Hz}, 3 \mathrm{H}, \mathrm{CH}_{3}\right), 1.24\left(\mathrm{~s}, 9 \mathrm{H}, \mathrm{CH}_{3}\right)$; ${ }^{13} \mathrm{C} \mathrm{NMR}\left(100 \mathrm{MHz}, \mathrm{CDCl}_{3}\right) \delta: 194.1,176.5,167.1,164.1$, $163.6,151.6,141.5,134.6,132.6,130.9,130.3,129.5$, $128.6,127.9,127.7,127.4,126.8,124.7,113.6,109.8$, $62.8,61.6,57.4,55.4,55.0,43.6,30.8,14.1$; IR (KBr) $v$ : 3429, 2971, 1722, 1672, 1647, 1598, 1512, 1485, 1458, $1430,1351,1270,1229,1173,1151,1101,1023,978,888$, $844,811,792,741,694,669,630,585,551,506 \mathrm{~cm}^{-1}$; HRMS (ESI) calcd for $\mathrm{C}_{34} \mathrm{H}_{34} \mathrm{ClN}_{2} \mathrm{O}_{5}[\mathrm{M}+\mathrm{H}]^{+}$585.2156, found 585.2158.

Ethyl 1'-benzyl-5'-chloro-2-(4-chlorobenzoyl)-5-(cyclohexylamino)-2'-oxospiro[cyclopentane-1,3'-indoline]-2,4diene-3-carboxylate $(\mathbf{3 h})$ : Yellow solid, 76\% yield. m.p. $166 \sim 168{ }^{\circ} \mathrm{C} ;{ }^{1} \mathrm{H}$ NMR $\left(400 \mathrm{MHz}, \mathrm{CDCl}_{3}\right) \delta: 9.93(\mathrm{~d}, J=$ $7.2 \mathrm{~Hz}, 1 \mathrm{H}, \mathrm{NH}), 7.59$ (s, 1H, ArH), $7.34 \sim 7.28(\mathrm{~m}, 4 \mathrm{H}$, $\operatorname{ArH}), 7.02 \sim 6.98(\mathrm{~m}, 3 \mathrm{H}, \operatorname{ArH}), 6.82(\mathrm{~d}, J=2.0 \mathrm{~Hz}, 1 \mathrm{H}$, $\operatorname{ArH}), 6.65(\mathrm{~d}, J=8.4 \mathrm{~Hz}, 2 \mathrm{H}, \operatorname{ArH}), 6.25(\mathrm{~d}, J=8.4 \mathrm{~Hz}$, $1 \mathrm{H}, \mathrm{CH}), 5.02\left(\mathrm{~d}, J=15.6 \mathrm{~Hz}, 1 \mathrm{H}, \mathrm{CH}_{2}\right), 4.14 \sim 4.06(\mathrm{~m}$, $\left.2 \mathrm{H}, \mathrm{CH}_{2}\right), 3.85 \sim 3.77\left(\mathrm{~m}, 1 \mathrm{H}, \mathrm{CH}_{2}\right), 371(\mathrm{~d}, J=15.6 \mathrm{~Hz}$, $\left.1 \mathrm{H}, \mathrm{CH}_{2}\right), 3.65 \sim 3.62(\mathrm{~m}, 1 \mathrm{H}, \mathrm{CH}), 1.89 \sim 1.87(\mathrm{~m}, 2 \mathrm{H}$, $\left.\mathrm{CH}_{2}\right), 1.71 \sim 1.67\left(\mathrm{~m}, 1 \mathrm{H}, \mathrm{CH}_{2}\right), 1.51 \sim 1.46\left(\mathrm{~m}, 2 \mathrm{H}, \mathrm{CH}_{2}\right)$, $1.33 \sim 1.26\left(\mathrm{~m}, 3 \mathrm{H}, \mathrm{CH}_{2}\right), 0.88\left(\mathrm{t}, J=6.8 \mathrm{~Hz}, 3 \mathrm{H}, \mathrm{CH}_{3}\right)$; ${ }^{13} \mathrm{C} \mathrm{NMR}\left(100 \mathrm{MHz}, \mathrm{CDCl}_{3}\right) \delta: 189.3,173.5,163.7,160.9$, $147.1,142.6,139.4,136.3,135.1,133.8,129.5,128.6$, $128.2,127.8,127.7,127.5,126.7,122.8,110.7,110.1$, $61.3,54.2,44.8,34.2,33.8,25.1,24.5,13.7$; IR (KBr) v: 3428, 2968, 1688, 1664, 1621, 1578, 1496, 1489, 1432, 1366, 1285, 1206, 1165, 1131, 1087, 1024, 992, 863, 838, $809,787,744,702,668,627,558,536,505 \mathrm{~cm}^{-1}$; HRMS (ESI) calcd for $\mathrm{C}_{35} \mathrm{H}_{33} \mathrm{Cl}_{2} \mathrm{~N}_{2} \mathrm{O}_{4}[\mathrm{M}+\mathrm{H}]^{+}$615.1817, found 615.1812 .
4.4 General procedure for the synthesis of functionalized oxindolin-2-ones $(\mathbf{4 a} \sim \mathbf{4 e})$

A mixture of alkylisocyanide $(0.30 \mathrm{mmol})$, DMAD $(0.30 \mathrm{mmol})$ and 3 -arylideneindolin-2-one $(0.3 \mathrm{mmol})$ in toluene $(10.0 \mathrm{~mL})$ was stirred at room temperature for $4 \mathrm{~h}$. Then, the solvent was removed by rotatory evaporation at reduced pressure. The residue was subjected to column chromatography with petroleum ether and ethyl acetate $(V: V=4: 1)$ as eluent to give the pure product.

Dimethyl (Z)-2-((tert-butylimino)methylene)-3-(3-(4methoxybenzylidene)-2-oxoindolin-1-yl)succinate (4a): Yellow solid, $67 \%$ yield. m.p. $164 \sim 166{ }^{\circ} \mathrm{C} ;{ }^{1} \mathrm{H}$ NMR $\left(400 \mathrm{MHz}, \mathrm{CDCl}_{3}\right) \delta: 8.39(\mathrm{~d}, J=8.8 \mathrm{~Hz}, 2 \mathrm{H}, \mathrm{ArH}), 7.51$ $(\mathrm{d}, J=7.6 \mathrm{~Hz}, 1 \mathrm{H}, \mathrm{ArH}), 7.48(\mathrm{~s}, 1 \mathrm{H}, \mathrm{CH}), 7.24 \sim 7.23(\mathrm{~m}$, $1 \mathrm{H}, \mathrm{ArH}), 7.06 \sim 7.01(\mathrm{~m}, 2 \mathrm{H}, \mathrm{ArH}), 6.94 \sim 6.92(\mathrm{~m}, 2 \mathrm{H}$, $\mathrm{ArH}), 5.91(\mathrm{~s}, 1 \mathrm{H}, \mathrm{CH}), 3.86\left(\mathrm{~s}, 3 \mathrm{H}, \mathrm{OCH}_{3}\right), 3.74(\mathrm{~s}, 3 \mathrm{H}$, $\left.\mathrm{OCH}_{3}\right), 3.70\left(\mathrm{~s}, 3 \mathrm{H}, \mathrm{OCH}_{3}\right), 1.37\left(\mathrm{~s}, 9 \mathrm{H}, \mathrm{CH}_{3}\right) ;{ }^{13} \mathrm{C} \mathrm{NMR}$ $\left(100 \mathrm{MHz}, \mathrm{CDCl}_{3}\right) \delta: 170.1,168.7,165.7,164.7,161.6$, $139.9,137.5,134.6,128.2,127.0,124.9,122.7,121.8$, $118.5,113.7,109.2,62.3,61.1,55.4,52.9,51.8,51.0,29.9$; IR (KBr) v: 3437, 3088, 2951, 2841, 2074, 1753, 1664, $1586,1555,1508,1469,1433,1383,1315,1262,1174$, 1107, 1022, 921, 845, 783, 747, 677, 645, 596, $546 \mathrm{~cm}^{-1}$; HRMS (ESI) calcd for $\mathrm{C}_{27} \mathrm{H}_{28} \mathrm{~N}_{2} \mathrm{NaO}_{6} \quad[\mathrm{M}+\mathrm{Na}]$ 499.1845, found 499.1845.

Dimethyl (Z)-2-((tert-butylimino)methylene)-3-(3-(4chlorobenzylidene)-2-oxoindolin-1-yl)succinate (4b): Yellow solid, 79\% yield. m.p. 168 $170{ }^{\circ} \mathrm{C} ;{ }^{1} \mathrm{H}$ NMR (400 $\left.\mathrm{MHz} \mathrm{CDCl}_{3}\right) \delta: 7.73$ (s, $\left.1 \mathrm{H}, \mathrm{ArH}\right), 7.57$ (d, $J=8.4 \mathrm{~Hz}, 3 \mathrm{H}$, $\mathrm{ArH}, \mathrm{CH}), 7.44$ (d, $J=8.4 \mathrm{~Hz}, 2 \mathrm{H}, \mathrm{ArH}), 7.29$ (d, $J=7.6$ $\mathrm{Hz}, 1 \mathrm{H}, \mathrm{ArH}), 7.07$ (d, $J=7.6 \mathrm{~Hz}, 1 \mathrm{H}, \mathrm{ArH}), 6.89$ (t, $J=$ $7.6 \mathrm{~Hz}, 1 \mathrm{H}, \mathrm{ArH}), 5.84(\mathrm{~s}, 1 \mathrm{H}, \mathrm{CH}), 3.76\left(\mathrm{~s}, 3 \mathrm{H}, \mathrm{OCH}_{3}\right)$, $3.71\left(\mathrm{~s}, 3 \mathrm{H}, \mathrm{OCH}_{3}\right), 1.45\left(\mathrm{~s}, 9 \mathrm{H}, \mathrm{CH}_{3}\right) ;{ }^{13} \mathrm{C} \mathrm{NMR}(100$ $\left.\mathrm{MHz}, \mathrm{CDCl}_{3}\right) \delta: 170.1,168.3,167.7,164.1,142.5,135.7$, $133.8,131.9,130.8,130.2,127.2,123.7,122.8,121.9$, $120.9,109.8,62.5,60.6,53.1,51.8,51.5,30.0$; IR (KBr) $v$ : 3684, 3081, 2983, 2949, 2063, 1740, 1687, 1593, 1465, 1370, 1311, 1264, 1179, 1104, 1018, 919, 868, 752, 706, $671,601,539 \mathrm{~cm}^{-1}$; HRMS (ESI) calcd for $\mathrm{C}_{26} \mathrm{H}_{25} \mathrm{ClN}_{2-}$ $\mathrm{NaO}_{5}[\mathrm{M}+\mathrm{Na}]^{+}$503.1350, found 503.1344.

Dimethyl (Z)-2-(3-(4-bromobenzylidene)-2-oxoindolin1-yl)-3-((tert-butylimino)methylene)succinate (4c): Yellow solid, 75\% yield. m.p. $177 \sim 179{ }^{\circ} \mathrm{C} ;{ }^{1} \mathrm{H}$ NMR (400 MHz, $\left.\mathrm{CDCl}_{3}\right) \delta: 7.71(\mathrm{~s}, 1 \mathrm{H}, \mathrm{ArH}), 7.61 \sim 7.56(\mathrm{~m}, 3 \mathrm{H}, \mathrm{ArH}$, $\mathrm{CH}), 7.50(\mathrm{~d}, J=8.4 \mathrm{~Hz}, 2 \mathrm{H}, \mathrm{ArH}), 7.30 \sim 7.28(\mathrm{~m}, 1 \mathrm{H}$, ArH), 7.07 (d, $J=8.0 \mathrm{~Hz}, 1 \mathrm{H}, \mathrm{ArH}), 6.88$ (t, $J=8.0 \mathrm{~Hz}$, $1 \mathrm{H}, \mathrm{ArH}), 5.83(\mathrm{~s}, 1 \mathrm{H}, \mathrm{CH}), 3.76\left(\mathrm{~s}, 3 \mathrm{H}, \mathrm{OCH}_{3}\right), 3.71(\mathrm{~s}$, $\left.3 \mathrm{H}, \mathrm{OCH}_{3}\right), 1.45\left(\mathrm{~s}, 9 \mathrm{H}, \mathrm{CH}_{3}\right) ;{ }^{13} \mathrm{C} \mathrm{NMR}(100 \mathrm{MHz}$, $\left.\mathrm{CDCl}_{3}\right) \delta: 170.1,168.3,167.7,164.1,142.5,135.7,135.4$, $133.3,130.6,130.2,128.9,127.1,122.7,121.9,120.9$, $109.8,62.5,60.7,53.0,51.8,51.5,30.0$; IR (KBr) v: 3685, 3069, 2947, 2063, 1740, 1687, 1593, 1467, 1370, 1310, 1262, 1179, 1112, 1071, 1014, 919, 868, 830, 751, 702, $668,601,542 \mathrm{~cm}^{-1}$; HRMS (ESI) calcd for $\mathrm{C}_{26} \mathrm{H}_{25} \mathrm{Br}-$ $\mathrm{N}_{2} \mathrm{NaO}_{5}[\mathrm{M}+\mathrm{Na}]^{+}$547.0845, found 547.0842.

Dimethyl (Z)-2-((tert-butylimino)methylene)-3-(3-(3chlorobenzylidene)-2-oxoindolin-1-yl)succinate (4d): Yel- 
low solid, $68 \%$ yield. m.p. $171 \sim 173{ }^{\circ} \mathrm{C} ;{ }^{1} \mathrm{H}$ NMR (400 $\left.\mathrm{MHz}, \mathrm{CDCl}_{3}\right) \delta: 7.72(\mathrm{~s}, 1 \mathrm{H}, \mathrm{ArH}), 7.60(\mathrm{~s}, 1 \mathrm{H}, \mathrm{CH})$, $7.55 \sim 7.50(\mathrm{~m}, 2 \mathrm{H}, \mathrm{ArH}), 7.40(\mathrm{~s}, 2 \mathrm{H}, \mathrm{ArH}), 7.30 \sim 7.27$ (m, 1H, ArH), 7.08 (d, J=7.6 Hz, 1H, ArH), 6.89 (t, $J=$ $7.2 \mathrm{~Hz}, 1 \mathrm{H}, \mathrm{ArH}), 5.84$ (s, 1H, CH), 3.77 (s, 3H, $\mathrm{OCH}_{3}$, $3.71\left(\mathrm{~s}, 3 \mathrm{H}, \mathrm{OCH}_{3}\right), 1.45\left(\mathrm{~s}, 9 \mathrm{H}, \mathrm{CH}_{3}\right) ;{ }^{13} \mathrm{C} \mathrm{NMR}(100$ $\left.\mathrm{MHz}, \mathrm{CDCl}_{3}\right) \delta: 170.1,168.3,167.6,164.1,142.6,136.7$, $135.3,134.6,130.3,129.9,129.4,128.9,127.7,127.2$, $122.9,122.1,120.8,109.8,62.5,60.6,53.1,51.8,51.5$, 30.0; IR (KBr) v: 3675, 3323, 2974, 2894, 2071, 1924, $1751,1705,1603,1564,1455,1380,1265,1186,1084$, 1050, 882, 784, 679, $603 \mathrm{~cm}^{-1}$; HRMS (ESI) calcd for $\mathrm{C}_{26} \mathrm{H}_{25} \mathrm{ClN}_{2} \mathrm{NaO}_{5}[\mathrm{M}+\mathrm{Na}]^{+}$503.1350, found 503.1347.

Dimethyl (Z)-2-(3-(4-chlorobenzylidene)-2-oxoindolin1-yl)-3-((cyclohexylimino)methylene)succinate (4e): Yellow solid, $77 \%$ yield. m.p. $181 \sim 183{ }^{\circ} \mathrm{C} ;{ }^{1} \mathrm{H}$ NMR $(400$ $\left.\mathrm{MHz}, \mathrm{CDCl}_{3}\right) \delta: 7.73$ (s, $\left.1 \mathrm{H}, \mathrm{ArH}\right), 7.57$ (d, $J=8.0 \mathrm{~Hz}, 3 \mathrm{H}$, ArH, CH), 7.44 (d, $J=8.4 \mathrm{~Hz}, 2 \mathrm{H}, \operatorname{ArH}), 7.30 \sim 7.28(\mathrm{~m}$, 1H, ArH), 7.08 (d, $J=8.0 \mathrm{~Hz}, 1 \mathrm{H}, \mathrm{ArH}), 6.89$ (t, $J=7.2$ $\mathrm{Hz}, 1 \mathrm{H}, \mathrm{ArH}), 5.85$ (s, 1H, CH), 3.89 (s, 1H, CH), 3.76 (s, $\left.3 \mathrm{H}, \mathrm{OCH}_{3}\right), 3.70\left(\mathrm{~s}, 3 \mathrm{H}, \mathrm{OCH}_{3}\right), 2.07 \sim 1.97\left(\mathrm{~m}, 2 \mathrm{H}, \mathrm{CH}_{2}\right)$, $1.77\left(\mathrm{~s}, 2 \mathrm{H}, \mathrm{CH}_{2}\right), 1.68 \sim 1.63\left(\mathrm{~m}, 2 \mathrm{H}, \mathrm{CH}_{2}\right), 1.37 \sim 1.32$ $\left(\mathrm{m}, 3 \mathrm{H}, \mathrm{CH}_{2}\right), 1.26\left(\mathrm{~s}, 1 \mathrm{H}, \mathrm{CH}_{2}\right) ;{ }^{13} \mathrm{C} \mathrm{NMR}(100 \mathrm{MHz}$, $\left.\mathrm{CDCl}_{3}\right) \delta: 170.1,168.4,167.8,163.8,142.5,135.7,135.4$, $133.3,130.6,130.1,128.9,127.2,122.7,121.9,120.9$, 109.7, 77.3, 77.0, 76.7, 60.7, 59.1, 53.1, 51.7, 51.6, 33.1, 33.0, 25.2, 23.9; IR (KBr) v: 2935, 2852, 2073, 1750, 1708, 1683, 1634, 1605, 1465, 1437, 1401, 1369, 1302, 1261, 1190, 1110, 1010, 882, 836, 747, 716, 675, 609, 583, $541 \mathrm{~cm}^{-1}$; HRMS (ESI) calcd for $\mathrm{C}_{28} \mathrm{H}_{27} \mathrm{ClN}_{2} \mathrm{NaO}_{5}[\mathrm{M}+$ $\mathrm{Na}]^{+}$529.1506, found 529.1503.

Supporting Information The copies of the ${ }^{1} \mathrm{H}$ NMR, and ${ }^{13} \mathrm{C}$ NMR spectra for all new products. The Supporting Information is available free of charge via the Internet at http://sioc-journal.cn

\section{References}

[1] (a) Yang, Y. T.; Zhu, J. F., Liao, G. C.; Xu, H. J.; Yu, B. Med. Chem. 2018, 25, 2233.

(b) Ye, N.; Chen, H. Y.; Wold, E. A.; Shi, P. Y.; Zhou, J. ACS Infect. Dis. 2016, 2, 382.

(c) Yu, B.; Yu, D. Q.; Liu, H. M. Eur. J. Med. Chem. 2015, 97, 673.

(d) Yu, B.; Yu, Z. Q.; Qi, P. P.; Yu, D. Q.; Liu, H. M. Eur. J. Med. Chem. 2015, 95, 35.

(e) Galliford, C. V.; Scheidt, K. A. Angew. Chem., Int. Ed. 2007, 46, 8748 .

[2] (a) Singh, G. S.; Desta, Z. Y. Chem. Rev. 2012, 112, 6104 (b) Hong, L.; Wang, R. Adv. Synth. Catal. 2013, 355, 1023. (c) Liu, Y.; Wang, H.; Wan, J. P. Asian, J. J. Org. Chem. 2013, 2, 374.

(d) Abdukader, A.; Zhang, Y. H.; Zhang, Z. P.; Liu, C. J. Chin. J. Org. Chem. 2016, 36, 875 (in Chinese).

(阿布力米提·阿布都卡德尔, 张永红, 张增鹏, 刘晨江, 有机化 学, 2016, 36, 875.)

(e) Lin, Y.; Du, D. M. Chin. J. Org. Chem. 2020, 40, 3214 (in Chinese).

(林晔, 杜大明, 有机化学, 2020, 40, 3214.)

(f) Zhao, S.; Li, C. P.; Xu, B.; Liu, H. Chin. J. Org. Chem. 2020, 40, 1549 (in Chinese).
(赵森, 李淳朴, 许斌, 柳红, 有机化学, 2020, 40, 1549.)

(g) Zhang, D. Q.; Liua, X.; Pang, X. J.; Liu, H. M.; Zhang, Q. R. Chin. J. Org. Chem. 2021, 41, 267 (in Chinese).

(张丹青, 柳旭, 庞晓静, 刘宏民, 张秋荣, 有机化学, 2021，41, 267.)

[3] (a) Cheng, D. Q.; Ishihara, Y.; Tan, B.; Barbas, C. F. ACS Catal. 2014, 4, 743 .

(b) Mei, G. J.; Shi, F. Chem. Commun. 2018, 54, 6607

(c) Boddy, A. J.; Bull, J. A. Org. Chem. Front 2021, 8, 1026.

(d) Luo, J. H.; Geng, W. S.; Cao, S. X.; He, Z. J. Chin. J. Org Chem. 2020, 40, 40 (in Chinese).

(罗京华, 耿玮笙, 曹仕轩, 贺峥杰, 有机化学, 2020, 40, 40.)

(e) Guo, X.; Guo, Y. J.; Kong, D. Z.; Lu, H. J.; Hua, Y. Z.; Wang, M. C. Chin. J. Org. Chem. 2020, 40, 1999 (in Chinese).

(郭欣, 郭亚军, 孔德志, 卢会杰, 华远照, 王敏灿, 有机化学, 2020, 40, 1999.)

[4] (a) Zhu, J. Eur. J. Org. Chem. 2003, 1133.

(b) Lygin, A. V.; Meijere, A. Angew. Chem., Int. Ed. 2010, 49, 9094. (c) Gulevich, A. V.; Zhdanko, A. G.; Orru, Romano V. A.; Nenajdenko, Valentine G. Chem. Rev. 2010, 110, 5235.

[5] (a) Dömling, A; Huang, Y. J. Synthesis 2010, 17, 2859. (b) Dömling, A.; Ugi, I. Angew. Chem., Int. Ed. 2000, 39, 3168.

(c) Wang, W.; Dömling, A. J. Comb. Chem. 2009, 11, 403.

[6] (a) Nair, V.; Rajesh, C.; Vinod, A. U.; Bindu, S.; Sreekanth, A. R.; Mathen, J. S.; Balagopal, L. Acc. Chem. Res. 2003, 36, 899. (b) Qiu, G.; Ding, Q.; Wu, J. Chem. Soc. Rev. 2013, 42, 5257.

[7] (a) Estevez, V.; Villacampa, M.; Menendez, J. C. Chem. Soc. Rev. 2014, 43, 4633 .

(b) Wan, J. P.; Liu, Y. RSC Adv. 2012, 2, 9763.

(c) Wang, F.; Wei, T. Q.; Xu, P.; Wang, S. Y.; Ji, S. J. Chin. Chem. Lett. 2019, 30, 379.

(d) Yan, L. J.; Yan, Y. X.; Chen, X. B.; Wang Y. C. Chin. J. Org. Chem. 2020, 40, 856 (in Chinese).

(严丽君，问玉金鍂，雪冰，王永超，有机化学, 2020, 40, 856.)

(e) Wang, Y. Y.; Liu, Y. Y. Acta Chim. Sinica 2019, 77, 418 (in Chinese).

(王昱望，刘云云，化学学报, 2019, 77, 418.)

(f) Yang, L.; Wan, J. P. Green Chem. 2020, 22, 3074.

(g) Liu, B. Y.; Xu, X. J.; Huang, L. L.; Feng, H. D. Chin. J. Org. Chem. 2020, 40, 1290 (in Chinese).

(刘博瑜，徐仙君，黄立梁，冯煌迪，有机化学, 2020, 40, 1290.)

[8] (a) Sadjadi, S.; Heravi, M. M.; Nazari, N. RSC Adv. 2016, 6, 53203. (b) Khan, I.; Zaib, S.; Ibrar, A. Org. Chem. Front. 2020, 7, 3734.

(c) Rostamnia, S. RSC Adv. 2015, 5, 97044.

[9] (a) Safaei, H. R.; Dehbozorgi, F. RSC Adv. 2016, 6, 26783.

(b) Wang, H. Y.; Bao, M.; Jiang, B.; Li, L. RSC $A d v$. 2016, 6, 6459.

(c) Gao, Q.; Hao, W. J.; Liu, F.; Tu, S. J.; Wang, S. L.; Li, G. G.; Jiang, B. Chem. Commun. 2016, 52, 900.

[10] (a) Yavari, I.; Arab-Salmanabadi, S.; Aminkhani, A. Chin. Chem. Lett. 2012, 23, 49

(b) Baharfar, R.; Jaafari, L.; Azimi, R. Chin. Chem. Lett. 2011, 22, 943.

(c) Mohtat, B.; Djahaniani, H.; Yavari, I.; Jam, S. A. Chin. Chem. Lett. 2011, 22, 771

(d) Adiba, M.; Mohammadia, B.; Sheikhia, E.; Bijanzadeh, H. R. Chin. Chem. Lett. 2011, 22, 314.

[11] (a) Neochoritis, C. G.; Stotani, S.; Misshra, B.; Dömling, A. Org. Lett. 2015, 17, 2002.

(b) Tang, T.; Fei, X. D.; Ge, Z. Y.; Chen, Z.; Zhu, Y. M.; Ji, S. J. J. Org. Chem. 2013, 78, 3170.

(c) Cao, M.; Fang, Y. L.; Wang, Y. C.; Xu, X. J.; Xi, Z. W.; Tang, S. ACS Comb. Sci. 2020, 22, 268.

[12] (a) Haung, J. Y.; Barve, I. J.; Sun, C. M. Org. Biomol. Chem. 2019, 17,3040 .

(b) Zakharova, E. A.; Shmatova, O. I.; Kutovaya, I. V.; Khrustalev V. N.; Nenajdenko, V. G. Org. Biomol. Chem. 2019, 17, 3433.

(c) Kurva, M.; Kerim, M. D.; Gamez-Montano, R.; El Kaim, L. 
Org. Biomol. Chem. 2019, 17, 9655.

(d) Xi, Z. W.; He, Y.; Liu, L. Q.; Wang, Y. C. Org. Biomol. Chem. 2020, 18,8089 .

[13] (a) Ramezanpour, S.; Rezaei, M. N.; Vaezghaemi, A.; Frank, R. New J. Chem. 2018, 42, 17533.

(b) Atar, A. B.; Kang, J. M.; Jadhav, A. H. New J. Chem. 2020, 44, 3241.

(c) Alavinia, S.; Ghorbani-Vaghei, R. New J. Chem. 2020, 44, 13062.

(d) Li, S. S.; Zeng, G.; Xing, X. Q.; Yang, Z. H.; Ma, F. Y.; Li, B. X.; Cheng, W. Y.; Zhang, J. K.; He, R. Y. New J. Chem. 2021, 45, 1834.

[14] (a) Sun, J.; Sun, Y.; Gong, H.; Xie, Y. J.; Yan, C. G. Org. Lett. 2012 , 14,5172 .

(b) Sun, J.; Xie, Y. J.; Yan, C. G. J. Org. Chem. 2013, 78, 8354.

(c) Gao, H.; Sun, J.; Yan, C. G. J. Org. Chem. 2014, 79, 4131.

(d) Han, Y.; Sheng, Y. J.; Yan, C. G. Org. Lett. 2014, 16, 2654.

(e) Sun, J.; Chen, L.; Gong, H.; Yan, C. G. Org. Biomol. Chem. 2015, 13, 5905.

(f) Chen, L.; Sun, J.; Xie, J.; Yan, C. G. Org. Biomol. Chem. 2016, 14, 6497.

[15] (a) Shi, R. G.; Yan, C. G. Chin. Chem. Lett. 2016, 27, 575.

(b) Wu, P.; Gao, H.; Sun, J.; Yan, C. G. Chin. Chem. Lett. 2017, 28, 329.

(c) Yang, R. Y.; Sun, J.; Tao, Y.; Sun, Q.; Yan, C. G. J. Org. Chem. 2017, 82, 13277. (d) Yang, R. Y.; Sun, J., Sun, Q.; Yan, C. G. J. Org. Chem. 2018, 83, 5909 .

(e) Wang, D. Q.; Sun, J.; Yan, C. G. ChemistrySelect 2019, 4, 10550 .

(f) Sun, J.; Yang, R. Y.; Zhan, S. C.; Yan, C. G. ChemistrySelect 2019, 4, 10100.

(g) Ye, R.; Yan, C. G. Eur. J. Org. Chem. 2019, 5882.

[16] (a) Sun, J.; Zhang, Y.; Zhang, R. G.; Yan, C. G. Org. Biomol. Chem. 2019, 17, 3978.

(b) Cao, J.; Yang, F.; Sun, J.; Huang, Y.; Yan, C. G. J. Org. Chem. 2019, 84, 622.

(c) Zhan, S. C.; Sun, J.; Liu, R. Z.; Yan, C. G. Org. Biomol. Chem. 2020, 18, 163.

(d) Zhu, M. J.; Han, Y.; Liu, C. Z.; Ma, W. Q.; Yan, C. G. Chin. Chem. Lett. 2020, 31, 1554.

(e) Sun, J.; Cao, J.; Han, Y.; Yan, C. G. Chin. J. Org. Chem. 2020, 40, 4122 (in Chinese).

(孙晶, 曹栓, 韩芗, 颜朝国, 有机化学, 2020, 40, 4122.)

[17] Xu, T. T.; Wang, S. Y.; Ji, S. J. Chin. J. Org. Chem. 2006, 26, 1414 (in Chinese).

(徐天图, 汪顺义, 纪顺俊, 有机化学, 2006, 26, 1414.)

[18] (a) Sarvary, A.; Shaabani, S.; Ghanji, N.; Shaabani, A. J. Sulfur Chem. 2015, 36, 117.

(b) Sarvary, A.; Shaabani, S.; Shaabani, A.; Ng, S. W. Tetrahedron Lett. 2011, 52, 5930.

(c) Alizadeh, A.; Ahmadi, M.; Monatsh. Chem. 2011, 142, 1249.

(Lu, Y.) 